\title{
Phase-Reduction Approach to Synchronization of Spatiotemporal Rhythms in Reaction-Diffusion Systems
}

\author{
Hiroya Nakao* \\ Graduate School of Information Science and Engineering, Tokyo Institute of Technology, \\ Tokyo 152-8552, Japan \\ Tatsuo Yanagita \\ Osaka Electro-Communication University, Neyagawa 572-8530, Japan
}

Yoji Kawamura

Department of Mathematical Science and Advanced Technology, Japan Agency for Marine-Earth Science and Technology, Yokohama 236-0001, Japan

(Received 26 September 2013; revised manuscript received 30 January 2014; published 22 May 2014)

\begin{abstract}
Reaction-diffusion systems can describe a wide class of rhythmic spatiotemporal patterns observed in chemical and biological systems, such as circulating pulses on a ring, oscillating spots, target waves, and rotating spirals. These rhythmic dynamics can be considered limit cycles of reaction-diffusion systems. However, the conventional phase-reduction theory, which provides a simple unified framework for analyzing synchronization properties of limit-cycle oscillators subjected to weak forcing, has mostly been restricted to low-dimensional dynamical systems. Here, we develop a phase-reduction theory for stable limit-cycle solutions of reaction-diffusion systems with infinite-dimensional state space. By generalizing the notion of isochrons to functional space, the phase-sensitivity function - a fundamental quantity for phase reduction - is derived. For illustration, several rhythmic dynamics of the FitzHugh-Nagumo model of excitable media are considered. Nontrivial phase-response properties and synchronization dynamics are revealed, reflecting their complex spatiotemporal organization. Our theory will provide a general basis for the analysis and control of spatiotemporal rhythms in various reaction-diffusion systems.
\end{abstract}

DOI: 10.1103/PhysRevX.4.021032

\section{INTRODUCTION}

Phase-reduction theory provides a general framework to simplify multidimensional ordinary differential equations (ODEs) describing weakly perturbed limit-cycle oscillators to one-dimensional approximate phase equations [1-5]. It has drastically facilitated theoretical and experimental analysis of the synchronization properties of weakly interacting nonlinear oscillators such as chemical oscillators and spiking neurons [1-9]. Methods for controlling limit-cycle oscillators have also been developed on the basis of phase-reduction theory [10-13].

In real-world systems, rhythmic dynamics often arise collectively from a number of spatially distributed interacting elements, rather than from a single isolated oscillator, e.g., heartbeats generated by an ensemble of pulsating cardiac cells [1,14-16]. Such systems are often modeled by reaction-diffusion (RD) systems, and the collective

*nakao@mei.titech.ac.jp

Published by the American Physical Society under the terms of the Creative Commons Attribution 3.0 License. Further distribution of this work must maintain attribution to the author(s) and the published article's title, journal citation, and DOI.
Subject Areas: Complex Systems, Nonlinear Dynamics

spatiotemporal rhythms are described by stable limit-cycle solutions of the RD systems [1,2,17-25]. Synchronization of collective spatiotemporal rhythms has been investigated experimentally in chemical systems [26,27] and may be of significant practical importance, e.g., in biomedical engineering [14-16]. In order to analyze and control the dynamics of collective spatiotemporal rhythms, it is desirable to develop a phase-reduction theory for the RD systems.

Various types of low-dimensional phase equations have been derived for RD systems, in particular, for traveling pulses $[2,24,25,28-34]$ and for rotating spirals [35,36]. In most cases, it is assumed that the system is symmetric with respect to continuous spatial translation or rotation and the spatial structure is rigidly translating or rotating, so that their location or angle is simply identified as the phase. However, such assumptions exclude various intriguing rhythmic dynamics of RD systems that lack continuous spatial symmetry. Since limit cycles are essentially associated with temporal translational symmetry, we should be able to derive phase equations from general RD systems without recourse to spatial symmetry.

Our goal in the present study is to develop, without assuming any spatial symmetry or rigidity, a 
phase-reduction theory for weakly perturbed RD systems exhibiting stable rhythmic dynamics. We solve this problem by generalizing the conventional phase-reduction theory for ODEs to RD systems. Our theory gives a systematic method to approximate rhythmic dynamics of RD systems with infinite-dimensional state space by onedimensional phase equations, thereby facilitating detailed analysis of the synchronization dynamics of rhythmic spatiotemporal patterns. As a simple example, we analyze mutual synchronization between two interacting layers of RD systems exhibiting rhythmic dynamics. The proposed theory provides a simple, unified description of rhythmic spatiotemporal patterns and will be the basis for developing methods to control and design rhythmic spatiotemporal patterns in RD systems.

This article is organized as follows: In Sec. II, essential results of the proposed phase-reduction theory for RD systems exhibiting spatiotemporal rhythms and its application to mutual synchronization of coupled RD systems are presented; details of the theory are given in Appendix B. Section III illustrates the theoretical results using several types of rhythmic spatiotemporal dynamics exhibited by the FitzHugh-Nagumo model of excitable media, and Sec. IV summarizes the results. Appendix A reviews the conventional phase-reduction theory for low-dimensional limit cycles described by ODEs, and Appendix B gives the full derivation of the phase-reduction theory for RD systems with emphasis on the notion of isochrons. Appendix $\mathrm{C}$ presents phase-sensitivity functions for the target waves, Appendix D shows nonlinear phase response of the RD systems to strong perturbations, Appendix E discusses frequencies of the synchronized patterns, and Appendix F gives details of Videos 1-6 showing synchronization dynamics of the FitzHugh-Nagumo model.

\section{PHASE DESCRIPTION OF SPATIOTEMPORAL RHYTHMS}

In this section, we summarize the essential results of the proposed phase-reduction theory for RD systems and apply it to mutual synchronization of a pair of coupled RD systems. Full derivation of the theory will be given in Appendix B. See also Appendix A for a review of the phase-reduction theory for low-dimensional limit-cycle oscillators described by ODEs.

\section{A. Phase reduction of limit-cycle solutions in reaction-diffusion systems}

We consider weakly perturbed RD systems exhibiting stable rhythmic dynamics, described by

$$
\frac{\partial}{\partial t} \mathbf{X}(\mathbf{r}, t)=\mathbf{F}(\mathbf{X}, \mathbf{r})+\mathrm{D} \nabla^{2} \mathbf{X}+\mathbf{p}(\mathbf{r}, t)
$$

Here, the vector field $\mathbf{X}(\mathbf{r}, t)$ represents the state (e.g., concentrations of chemical species) of the RD medium at point $\mathbf{r}$ at time $t, \mathbf{F}(\mathbf{X}, \mathbf{r})$ represents the local reaction dynamics at $\mathbf{r}, \mathrm{D} \nabla^{2} \mathbf{X}$ represents the diffusion of $\mathbf{X}$ over the medium with a matrix D of diffusion constants, and $\mathbf{p}(\mathbf{r}, t)$ represents weak spatiotemporal perturbations. Explicit dependence of $\mathbf{F}$ on $\mathbf{r}$, such as medium heterogeneity, may exist. We assume that the RD system (1) without perturbation $(\mathbf{p}=\mathbf{0})$ exhibits a stable rhythmic dynamics; i.e., it possesses a stable limit-cycle solution $\chi: \mathbf{X}_{0}(\mathbf{r}, t)=$ $\mathbf{X}_{0}(\mathbf{r}, t+T)$ of period $T=2 \pi / \omega$, where $\omega$ denotes frequency and that this solution persists and deforms only slightly even if the system is weakly perturbed $(\mathbf{p} \neq \mathbf{0})$. Such a limit cycle includes the circulating pulses on a ring, oscillating spots, target waves, and rotating spirals that we will analyze in Sec. III (see Figs. 1, 2, 3, and 4). Note that the frequency $\omega$ of the unperturbed pattern is strictly constant over space; i.e., every part of the system evolves consistently with the same $\omega$. The perturbation may slightly disturb the pattern and shift the overall frequency, but the whole system should maintain its consistency and not split into multiple regions with different frequencies.

The purpose of the phase-reduction theory is to derive a simple closed equation for the phase $\theta$ approximately describing limit-cycle oscillations of Eq. (1) under weak perturbation $(\mathbf{p} \neq \mathbf{0})$. As in the ODE case (see Appendix A), we first introduce a phase $\theta=\omega t$ $(\bmod 2 \pi)$ to a system state $\mathbf{X}_{0}(\mathbf{r}, t)$ on the limit cycle $\chi$ so that $\dot{\theta}(t)=\omega$ constantly holds, and we denote the system state as $\mathbf{X}_{0}(\mathbf{r} ; \theta)$ using the phase $\theta$. To perform phase reduction, we also need to assign a phase to a system state $\mathbf{X}(\mathbf{r}, t)$ that is not on the limit cycle $\chi$ but eventually converges to $\chi$, since the system state can deviate from $\chi$ because of perturbations. Specifically, we need a functional $\theta=\Theta\{\mathbf{X}(\mathbf{r}, t)\}$ that maps $\mathbf{X}(\mathbf{r}, t)$ in the basin of $\chi$ to a scalar phase $\theta$ such that $\dot{\theta}(t)=\omega$ constantly holds. This leads to the notion of isochrons $[1-5,37,38]$, i.e., equalphase contours of the system state around $\chi$. The notion of the isochrons is at the core of the conventional phasereduction theory for ODEs and should be generalized to RD systems. It is, however, generally impossible to obtain such a functional explicitly.

To proceed, we use the assumption that the perturbation is weak and focus on the vicinity of $\chi$. We make an ansatz that the phase $\Theta\{\mathbf{X}(\mathbf{r})\}$ of a system state $\mathbf{X}(\mathbf{r})$ near $\mathbf{X}_{0}(\mathbf{r} ; \theta)$ can be linearly approximated, using a certain function $\mathbf{Q}(\mathbf{r} ; \theta)$, as

$$
\Theta\{\mathbf{X}(\mathbf{r})\}=\theta+\left\langle\mathbf{Q}(\mathbf{r} ; \theta), \mathbf{X}(\mathbf{r})-\mathbf{X}_{0}(\mathbf{r} ; \theta)\right\rangle
$$

around $\chi$, where $\langle\mathbf{A}(\mathbf{r}), \mathbf{B}(\mathbf{r})\rangle=\int \mathbf{A}(\mathbf{r}) \cdot \mathbf{B}(\mathbf{r}) d \mathbf{r}$ is the inner product between two functions. For a system state $\mathbf{X}(\mathbf{r})=\mathbf{X}_{0}(\mathbf{r} ; \theta)$ on $\chi$ with phase $\theta$, an identity $\Theta\left\{\mathbf{X}_{0}(\mathbf{r} ; \theta)\right\}=\theta$ should hold by the above definition of the phase. Moreover, for any system state $\mathbf{X}(\mathbf{r}, t)$ near $\chi$ evolving under Eq. (1) with $\mathbf{p}=\mathbf{0}$, we require that 
$\theta(t)=\Theta\{\mathbf{X}(\mathbf{r}, t)\}$ satisfies $\dot{\theta}(t)=\omega$ constantly within a linear approximation.

As we will derive in Appendix B, if $\mathbf{Q}(\mathbf{r} ; \theta)$ is a periodic solution to a generalized adjoint equation

$$
\omega \frac{\partial}{\partial \theta} \mathbf{Q}(\mathbf{r} ; \theta)=-\mathrm{J}(\theta)^{\dagger} \mathbf{Q}(\mathbf{r} ; \theta)-\mathrm{D}^{\dagger} \nabla^{2} \mathbf{Q}(\mathbf{r} ; \theta)
$$

with a normalization condition

$$
\left\langle\mathbf{Q}(\mathbf{r} ; \theta), \frac{\partial \mathbf{X}_{0}(\mathbf{r} ; \theta)}{\partial \theta}\right\rangle=1
$$

the functional $\Theta\{\mathbf{X}(\mathbf{r})\}$ assumed in Eq. (2) satisfies the above requirements for the phase, and such a $\mathbf{Q}(\mathbf{r} ; \theta)$ plays the role of the phase-sensitivity function [1-5] for the RD system quantifying linear response characteristics of the system phase to weak perturbations. Here, $\mathrm{J}(\theta)=$ $\mathrm{J}\left[\mathbf{X}_{0}(\mathbf{r} ; \theta)\right]$ is a Jacobi matrix of $\mathbf{F}$ estimated at $\mathbf{X}=$ $\mathbf{X}_{0}(\mathbf{r} ; \theta)$ on $\chi$ and $\dagger$ indicates the matrix transpose.

Namely, we can show that the phase $\theta(t)=\Theta\{\mathbf{X}(\mathbf{r}, t)\}$ of the infinite-dimensional RD system (1) approximately obeys a simple one-dimensional phase equation

$$
\dot{\theta}(t)=\omega+\langle\mathbf{Q}(\mathbf{r} ; \theta), \mathbf{p}(\mathbf{r}, t)\rangle,
$$

which is correct up to the lowest order of the perturbation $\mathbf{p}(\mathbf{r}, t)$. Thus, once $\mathbf{Q}(\mathbf{r} ; \theta)$ is obtained from Eqs. (3) and (4), rhythmic dynamics of the RD system subjected to weak spatiotemporal perturbations, Eq. (1), can easily be analyzed using Eq. (5). This is the main result of the present study.

The phase equation (5) also shows that, if a system state $\mathbf{X}_{0}(\mathbf{r} ; \theta)$ with phase $\theta$ on $\chi$ is instantaneously perturbed by a weak spatial stimulus $\mathbf{s}(\mathbf{r})$, the response of the system phase after relaxation, namely, the phase-response curve (PRC) [1,5], is given by

$$
R(\theta)=\langle\mathbf{Q}(\mathbf{r} ; \theta), \mathbf{s}(\mathbf{r})\rangle
$$

within a linear approximation. This PRC $R(\theta)$ can be measured directly in numerical simulations by applying impulsive perturbations to the RD system, as we will illustrate in Sec. III.

\section{B. Mutual synchronization of spatiotemporal rhythms}

As a simple example of the phase-reduction approach, let us consider synchronization of a pair of weakly coupled RD systems exhibiting rhythmic dynamics,

$$
\begin{gathered}
\frac{\partial}{\partial t} \mathbf{X}_{1}(\mathbf{r}, t)=\mathbf{F}\left(\mathbf{X}_{1}, \mathbf{r}\right)+\mathrm{D} \nabla^{2} \mathbf{X}_{1}+\mathbf{G}\left\{\mathbf{X}_{1}, \mathbf{X}_{2}\right\}, \\
\frac{\partial}{\partial t} \mathbf{X}_{2}(\mathbf{r}, t)=\mathbf{F}\left(\mathbf{X}_{2}, \mathbf{r}\right)+\mathrm{D} \nabla^{2} \mathbf{X}_{2}+\mathbf{G}\left\{\mathbf{X}_{2}, \mathbf{X}_{1}\right\},
\end{gathered}
$$

where $\mathbf{X}_{1,2}$ represent the system states. We assume local and linear mutual coupling, $\mathbf{G}\{\mathbf{X}, \mathbf{Y}\}=\mathrm{K}[\mathbf{Y}(\mathbf{r}, t)-\mathbf{X}(\mathbf{r}, t)]$, with a diagonal matrix $\mathrm{K}$ representing the intensity of the weak mutual coupling. Experimental systems like Eq. (7) have been realized by coupling a pair of photosensitive BelousovZhabotinsky chemical reactions via video cameras and projectors [26], and by coupling a pair of electrochemical oscillators [27].

Denoting the phase variables of the two systems as $\theta_{1,2}$ and considering the coupling term $\mathbf{G}$ as weak perturbations, we can approximate Eq. (7) by a pair of coupled phase equations,

$\dot{\theta}_{1}(t)=\omega+\left\langle\mathbf{Q}\left(\mathbf{r} ; \theta_{1}\right), \mathbf{G}\left\{\mathbf{X}_{0}\left(\mathbf{r} ; \theta_{1}\right), \mathbf{X}_{0}\left(\mathbf{r} ; \theta_{2}\right)\right\}\right\rangle$,

$\dot{\theta}_{2}(t)=\omega+\left\langle\mathbf{Q}\left(\mathbf{r} ; \theta_{2}\right), \mathbf{G}\left\{\mathbf{X}_{0}\left(\mathbf{r} ; \theta_{2}\right), \mathbf{X}_{0}\left(\mathbf{r} ; \theta_{1}\right)\right\}\right\rangle$,

where $\mathbf{X}_{1,2}$ in $\mathbf{G}$ are approximated by $\mathbf{X}_{0}\left(\mathbf{r} ; \theta_{1,2}\right)$ as the lowest-order approximation [1,2]. Note that the two infinite-dimensional RD systems are reduced to just two one-dimensional phase equations.

The coupled phase equations (8) can be analyzed in the same way as those for ordinary limit cycles [1-5]. Since the coupling term $\mathbf{G}$ is small, we can apply the averaging approximation to Eqs. (8), which yields

$\dot{\theta}_{1}(t)=\omega+\Gamma\left(\theta_{1}-\theta_{2}\right), \quad \dot{\theta}_{2}(t)=\omega+\Gamma\left(\theta_{2}-\theta_{1}\right)$,

where the phase-coupling function $\Gamma$ is given by

$\Gamma(\phi)=\frac{1}{2 \pi} \int_{0}^{2 \pi}\left\langle\mathbf{Q}(\mathbf{r} ; \theta+\phi), \mathbf{G}\left\{\mathbf{X}_{0}(\mathbf{r} ; \theta+\phi), \mathbf{X}_{0}(\mathbf{r} ; \theta)\right\}\right\rangle d \theta$.

By subtraction, the phase difference $\phi=\theta_{1}-\theta_{2}$ obeys

$$
\dot{\phi}(t)=\Gamma(\phi)-\Gamma(-\phi)=\Gamma_{a}(\phi),
$$

where $\Gamma_{a}(\phi)$ is a $2 \pi$-periodic function. Here, $\Gamma_{a}(\phi)$ is antisymmetric because we consider two identical limit cycles (rhythmic patterns) with symmetric coupling.

Thus, the phase difference $\phi=\theta_{1}-\theta_{2}$ between the two RD systems approximately obeys a quite simple one-dimensional equation. By examining the zeros of $\Gamma_{a}(\phi)$ where $\dot{\phi}(t)=0$, we can predict the stationary phase differences at which phase synchronization occurs between the two limit-cycle orbits of the coupled RD systems under the phase-reduction approximation. The synchronized state with a stationary phase difference $\phi^{*}$ is linearly stable when $d \Gamma_{a}(\phi) / d \phi<0$ [unstable when $d \Gamma_{a}(\phi) / d \phi>0$ ] at $\phi=\phi^{*}$. A pair of stably synchronized RD systems with the phase difference $\phi^{*}$ undergoes phase-locked oscillations with a common frequency $\omega+\Gamma\left(\phi^{*}\right)$; i.e., their frequency shifts from the unperturbed value $\omega$ by $\Gamma\left(\phi^{*}\right)$. 
For symmetrically coupled identical RD systems, $\Gamma_{a}(\phi)$ always vanishes at $\phi=0$ and at $\phi= \pm \pi$, so the existence of in-phase $(\phi=0)$ and antiphase $(\phi= \pm \pi)$ synchronized states is assured.

\section{EXAMPLES}

In this section, we illustrate the phase-reduction theory for RD systems by numerical simulations. We analyze phase-response properties and synchronization dynamics of circulating pulses on a ring, oscillating spots, target waves, and rotating spirals of the FitzHugh-Nagumo model of excitable media (Figs. 1-4). Among these rhythmic patterns, the circulating pulses and rotating spirals are rigid and spatially symmetric, so they may, in principle, be

(a)

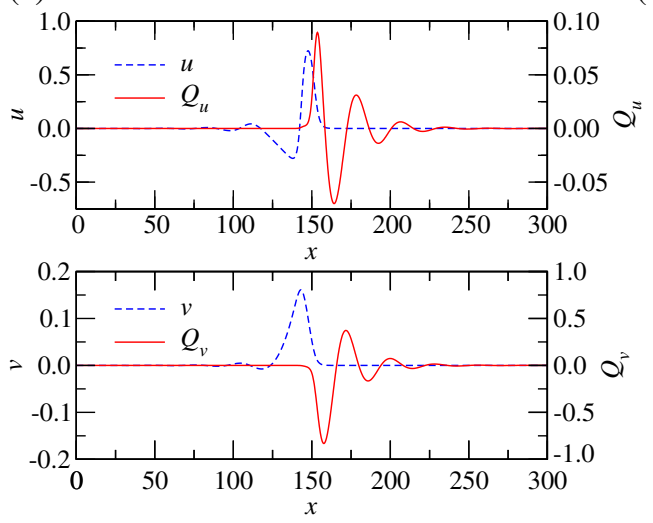

(c)

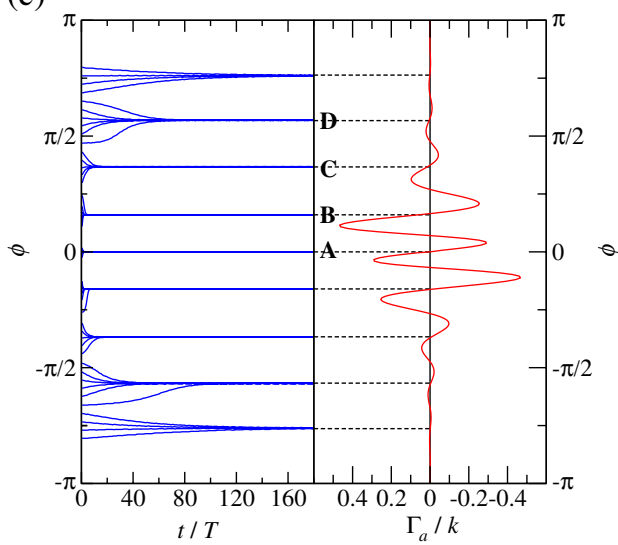

analyzed using the conventional methods [2,25,28-36]. In contrast, the oscillating spots and target waves are not rigid and lack translational or rotational symmetry; therefore, they cannot be treated by the conventional methods that rely on such assumptions. In any case, the phase reduction can provide a simple, unified approach to the synchronization properties of spatiotemporal rhythms. As we will see, complex spatiotemporal profiles of the rhythmic patterns can lead to interesting synchronization dynamics.

\section{A. The FitzHugh-Nagumo model}

The FitzHugh-Nagumo (FHN) reaction-diffusion model is a classical model of neural spike transmission, whose dynamics is described by

(b)
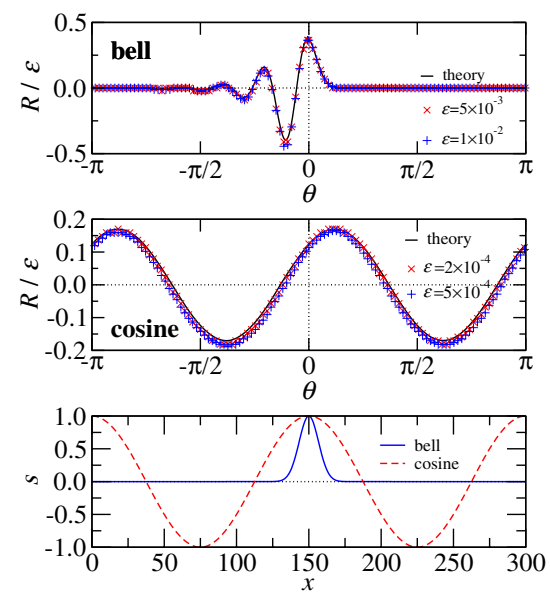

(d)

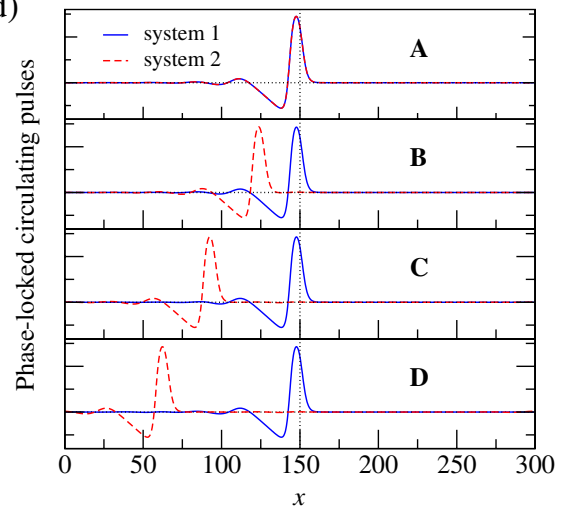

FIG. 1. Circulating pulses. The system is a $1 \mathrm{D}$ ring of length $L=300$ with periodic boundary conditions. The system parameters are $\alpha=0, \tau^{-1}=0.018, \gamma=1, \kappa=1$, and $\delta=0.02$. With these values, the pulse exhibits a wavy tail [19]. The oscillation period (time needed for the pulse to go around the ring) is $T \approx 551.8$. (a) Snapshots of the stable circulating pulse with a wavy tail, $\mathbf{X}_{0}(x ; \theta)=[u(x ; \theta), v(x ; \theta)]$, and the corresponding phase-sensitivity function, $\mathbf{Q}(x ; \theta)=\left[Q_{u}(x ; \theta), Q_{v}(x ; \theta)\right]$, for $\theta=0$. (b) Phase-response curves $R(\theta)$ of the circulating pulse normalized by the stimulus intensity $\varepsilon$. Either bell-shaped $\left[s(x)=\varepsilon \exp \left\{-(x-150)^{2} / 90\right\}\right]$ or cosine $[s(x)=\varepsilon \cos (4 \pi x / 300)]$ perturbation is given to the activator (u) component. Results obtained by direct numerical simulations with two different values of $\varepsilon$ are compared with the theory, $R(\theta)=\langle\mathbf{Q}(x ; \theta)$, $\mathbf{s}(x)\rangle$, where the inner product is taken over the 1D ring $(0 \leq x \leq L)$. (c) Evolution of the phase difference $\phi=\theta_{1}-\theta_{2}$ between two systems coupled through the $u$ component with the coupling intensity matrix $\mathrm{K}=\operatorname{diag}(k, 0)$ with $k=0.001$ and the antisymmetric part of the phasecoupling function $\Gamma_{a}(\phi)$ (rescaled by the coupling intensity $k$ ). Different blue lines show the evolution of $\phi$ for different initial conditions. The two pulses show multimodal phase locking. (d) Snapshots of phase-locked pulses with four different stable phase differences. Graphs (A-D) correspond to the stable phase differences shown in (c). 
(a)
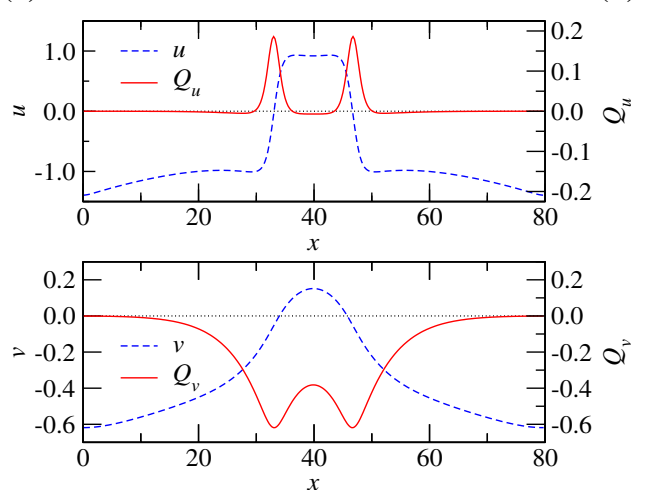

(c)
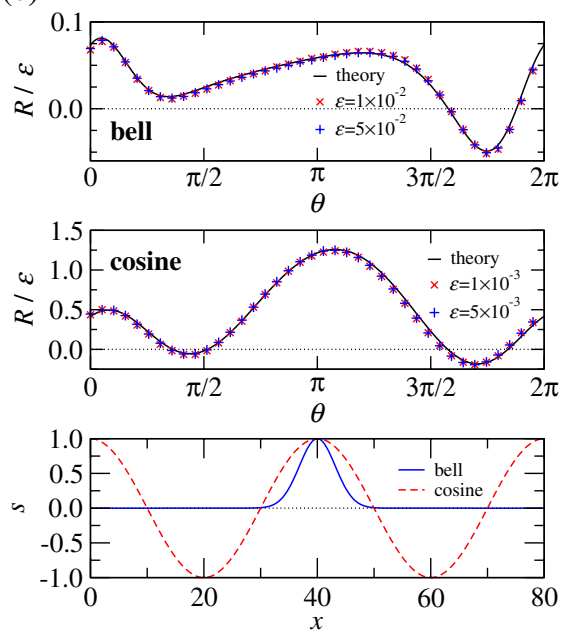

(d)

(e) (b)

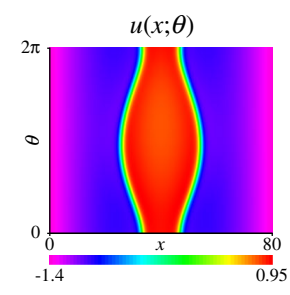

$v(x ; \theta)$

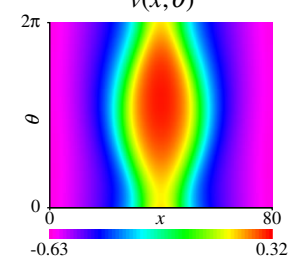

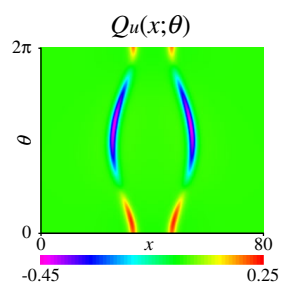

$Q_{v}(x ; \theta)$

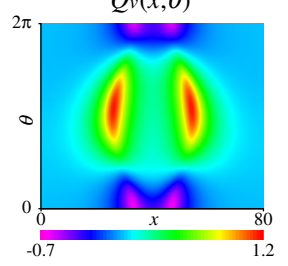

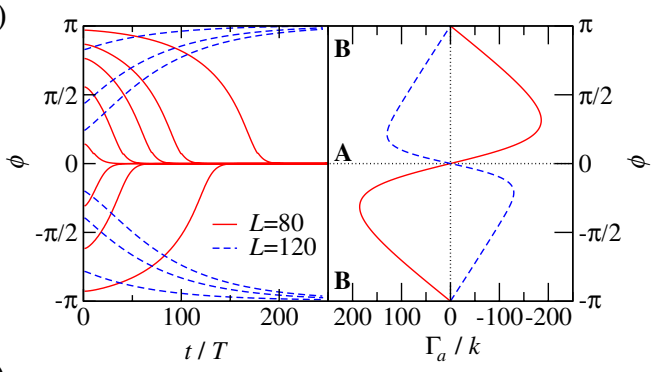
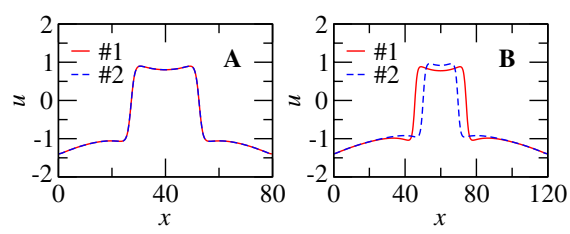

FIG. 2. Oscillating spots. The system is a 1D interval of length $L=80$ or $L=120$ with no-flux boundary conditions. The parameter $\alpha$ is space dependent; i.e., $\alpha(x)=\alpha_{0}+\left(\alpha_{1}-\alpha_{0}\right)(2 x / L-1)^{2}$ with $\alpha_{0}=-1.1$ and $\alpha_{1}=-1.6$ so that $\alpha$ is the largest at the center $(x=L / 2)$ and the smallest at the boundaries $(x=0, L)$. Other parameter values are $\tau^{-1}=0.03, \gamma=2.0, \kappa=1$, and $\delta=2.5$. With these conditions, an oscillating spot constrained at the center can be generated. The oscillation period is $T=194.8(L=80)$ or $T=204.0$ $(L=120)$. (a) Snapshots of the oscillating spot solution $\mathbf{X}_{0}(x)=[u(x), v(x)]$ and the corresponding phase-sensitivity function $\mathbf{Q}(x)=$ $\left[Q_{u}(x), Q_{v}(x)\right]$ for $\theta=0$. (b) Evolution of $\mathbf{X}_{0}(x ; \theta)$ and $\mathbf{Q}(x ; \theta)$ during $0 \leq \theta<2 \pi$. (c) Phase-response curves $R(\theta)$ of the oscillating spot normalized by the stimulus intensity $\varepsilon$. Perturbation $s(x)$ is either bell-shaped $\left[s(x)=\varepsilon \exp \left\{-4(x-L / 2)^{2} / L\right\}\right]$ or sinusoidal $[s(x)=\varepsilon \cos (4 \pi x / L)]$ and is given to the activator $(u)$ component. Results obtained by direct numerical simulations with two different values of $\varepsilon$ are compared with the theory, $R(\theta)=\langle\mathbf{Q}(x ; \theta), \mathbf{s}(x)\rangle$, where the inner product is taken over the 1D interval $(0 \leq x \leq L)$. (d) Evolution of phase differences between two systems coupled through the $u$ component with the intensity matrix $\mathrm{K}=\operatorname{diag}(k, 0)$ with $k=10^{-4}$, showing in-phase synchronization for $L=80$ (A) and antiphase synchronization for $L=120$ (B). Different lines show the evolution of $\phi$ for different initial conditions. The antisymmetric part of the phase-coupling function $\Gamma_{a}(\phi)$ (rescaled by the coupling intensity $k$ ) is shown for comparison. (e) Snapshots of activator patterns $u(x)$ of both systems in the in-phase (A) and antiphase (B) synchronized states.

$$
\begin{aligned}
& \mathbf{X}=\left(\begin{array}{l}
u \\
v
\end{array}\right), \quad \mathbf{F}=\left(\begin{array}{c}
u(u-\alpha)(1-u)-v \\
\tau^{-1}(u-\gamma v)
\end{array}\right), \\
& \mathrm{D}=\left(\begin{array}{ll}
\kappa & 0 \\
0 & \delta
\end{array}\right),
\end{aligned}
$$

where $u=u(\mathbf{r}, t)$ and $v=v(\mathbf{r}, t)$ are activator and inhibitor variables, respectively. By appropriately choosing the parameters $\alpha, \tau, \gamma$, and the diffusion constants $\kappa$ and $\delta$, the FHN model can exhibit various types of rhythmic spatiotemporal dynamics [19-22], such as the circulating pulses on a ring (Fig. 1), oscillating spots (Fig. 2), target waves (Fig. 3), and rotating spirals (Fig. 4).

In numerical simulations, the size of the system is $L=80-600$ for $1 \mathrm{D}$ cases, and it is discretized using $\Delta x=0.5-1.0$ spatial grids. For $2 \mathrm{D}$ cases, the system size is $L_{x} \times L_{y}=80 \times 80-120 \times 120$, and it is discretized with $\Delta x=\Delta y=0.5-1.0$ spatial grids. The explicit Euler method with a time step $\Delta t=0.01-0.05$ is used for time integration.

To numerically obtain the phase-sensitivity function $\mathbf{Q}(\mathbf{r} ; \theta)$, the adjoint equation (3) is integrated backward in time [5]. Namely, one period of the limit-cycle 
(a)

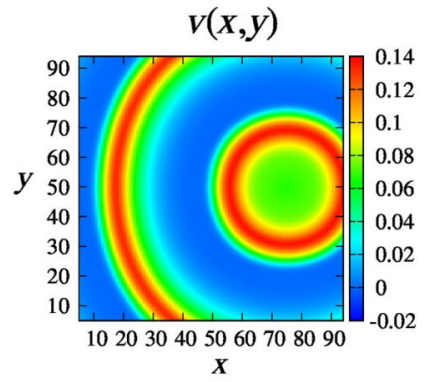

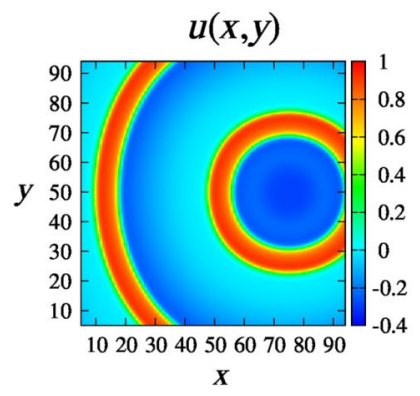
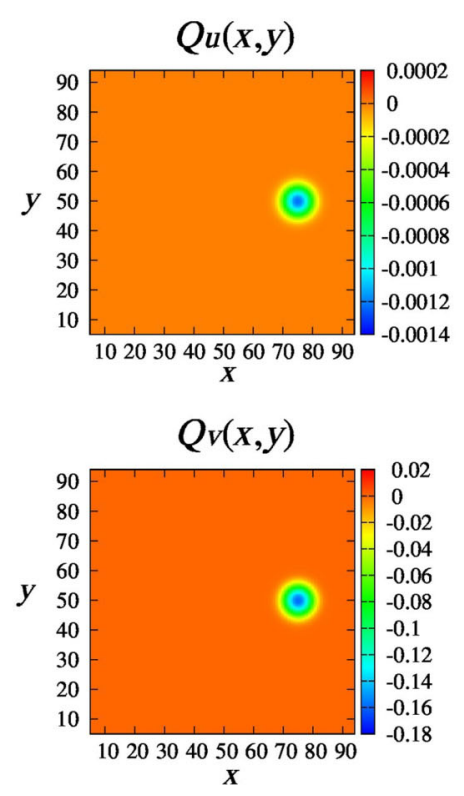

(c)

(d)
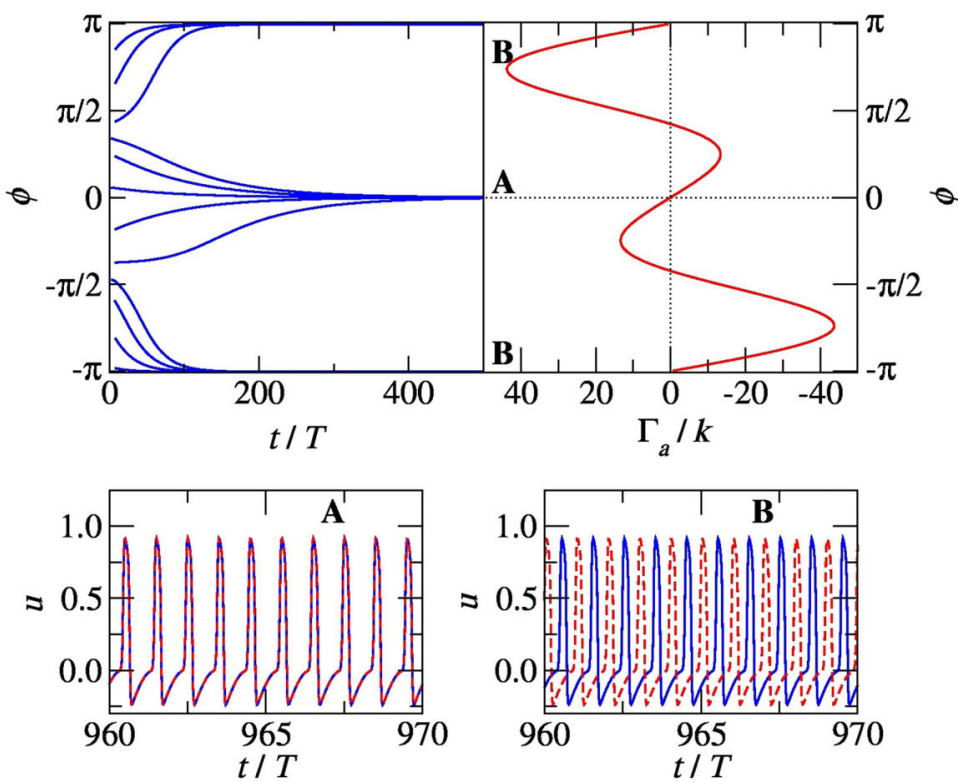

(b)
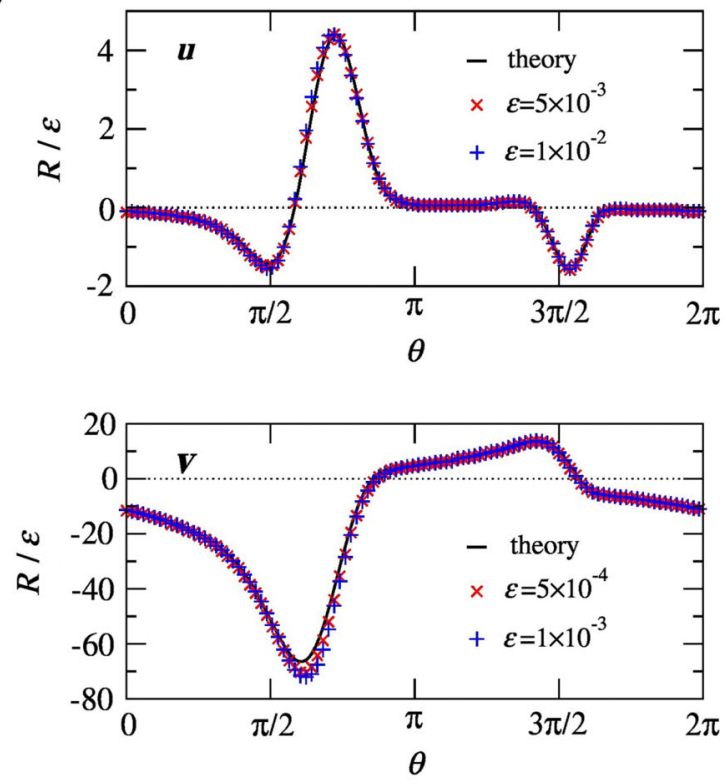

(e)

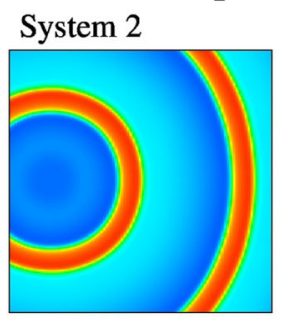

System 1

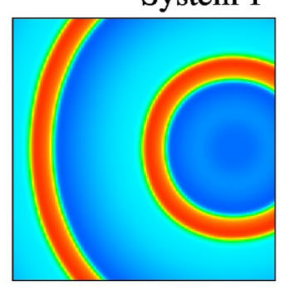

\section{Antiphase locking}

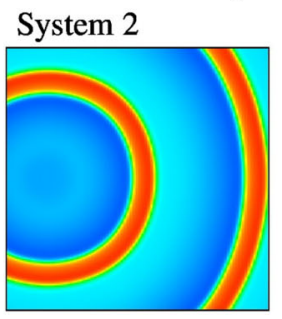

System 1

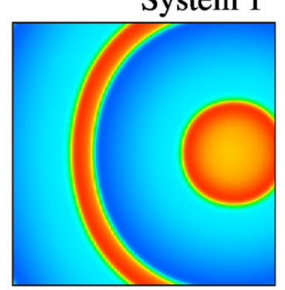

FIG. 3. Target waves. The system is a $2 \mathrm{D}$ square of side $L=100$ with no-flux boundary conditions. To create a pacemaker region, the parameter $\alpha$ is assumed to possess localized circular heterogeneity, i.e., $\alpha(x, y)=\alpha_{0}+\left(\alpha_{1}-\alpha_{0}\right) \exp \left(-r^{4} / r_{0}^{4}\right)$, where $r=\left[\left(x-x_{0}\right)^{2}+\right.$ $\left.\left(y-y_{0}\right)^{2}\right]^{1 / 2}$ is the distance from the pacemaker center at $\left(x_{0}, y_{0}\right)$ and $r_{0}$ is the radius of the pacemaker region, so $\alpha(x, y) \rightarrow \alpha_{1}$ as $r \rightarrow 0$ and $\alpha(x, y) \rightarrow \alpha_{0}$ as $r \rightarrow \infty$. The parameters are $\alpha_{0}=0.1, \alpha_{1}=-0.1, r_{0}=10$, and $\left(x_{0}, y_{0}\right)=(80,50)$. With these values, the system is self-oscillatory near the pacemaker center and is excitable otherwise. Other parameters are $\tau^{-1}=0.005, \gamma=2.5, \kappa=0.15$, and $\delta=0$. The temporal oscillation period is $T=205.4$. (a) Target-wave solution $\mathbf{X}_{0}(x, y)=[u(x, y), v(x, y)]$ and the corresponding phasesensitivity function $\mathbf{Q}(x, y)=\left[Q_{u}(x, y), Q_{v}(x, y)\right]$ at $\theta=0$ (see Appendix C for other values of $\theta$ ). (b) Phase-response curves $R(\theta)$ of the target wave normalized by the stimulus intensity $\varepsilon$. Sinusoidal perturbation $s(x, y)=\varepsilon \cos (4 \pi x / 100) \cos (4 \pi y / 100)$ is given either to the activator $(u)$ or inhibitor $(v)$ component. Results obtained by direct numerical simulations with two different values of $\varepsilon$ are compared with the theory, $R(\theta)=\langle\mathbf{Q}(x, y ; \theta), \mathbf{s}(x, y)\rangle$, where the inner product is taken over the 2D square $(0 \leq x, y \leq L)$. (c) Evolution of phase differences between two systems coupled through the $u$ component with the intensity matrix $\mathrm{K}=\operatorname{diag}\left(k=5 \times 10^{-4}, 0\right)$, compared with the antisymmetric part of the phase-coupling function $\Gamma_{a}(\phi)$ (rescaled by the coupling intensity $k$ ). Different blue lines show the evolution of $\phi$ for different initial conditions. Both in-phase (A) and antiphase (B) synchronization can occur, depending on the initial conditions. (d) Evolution of the activator $u$ measured at the center $[(x, y)=$ $(L / 2, L / 2)]$ of systems 1 and 2 in the in-phase (A) and antiphase (B) synchronized states. The solid line corresponds to system 1, and the dashed line corresponds to system 2. (e) Snapshots of the activator patterns $u(x, y)$ of both systems in the in-phase (A) and antiphase (B) synchronized states. 
(a)

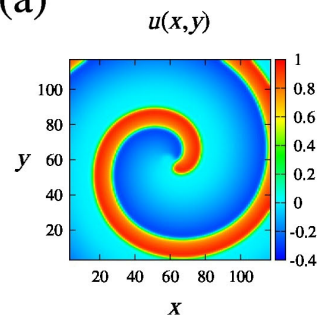

$v(x, y)$

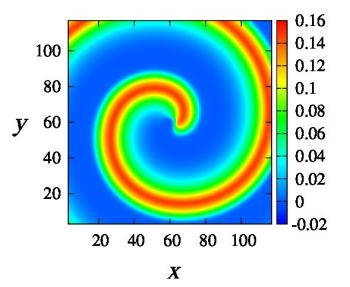

(c)
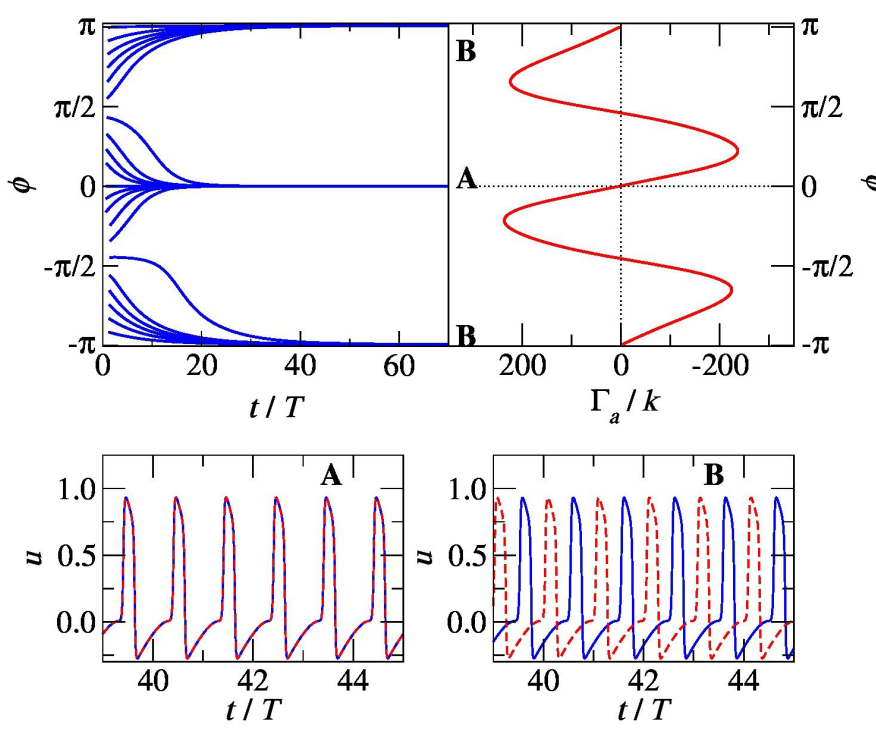
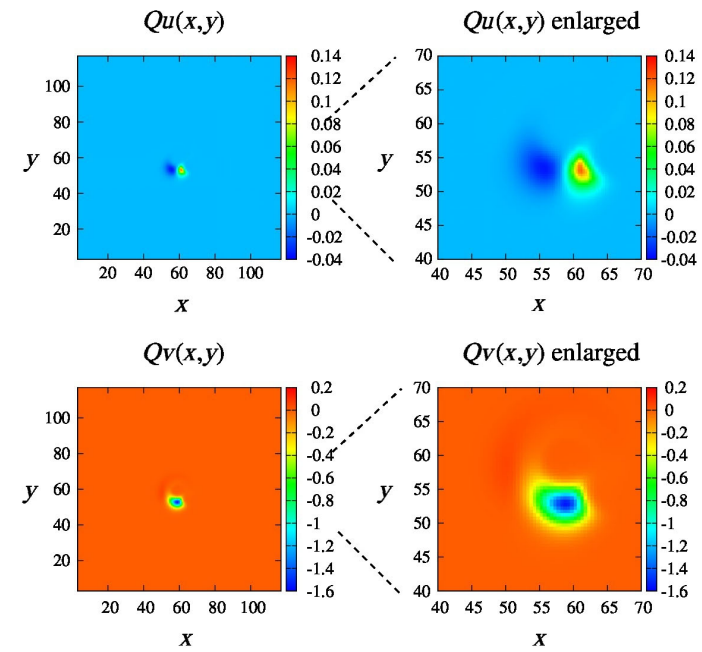

(d) (b)
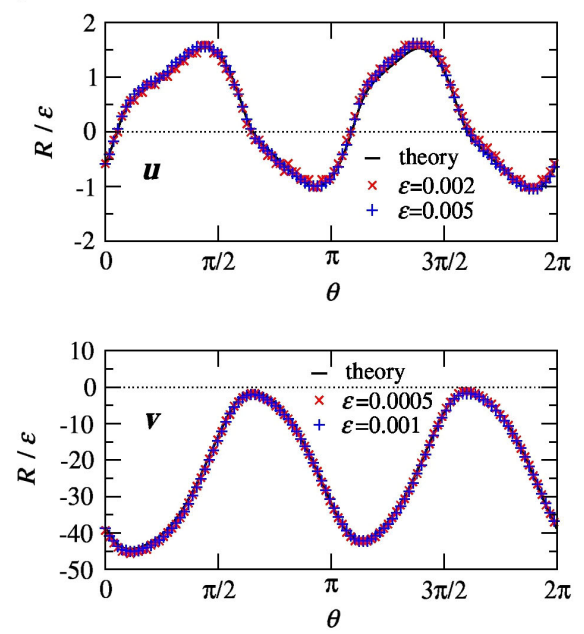

In-phase locking

System 1

System 2
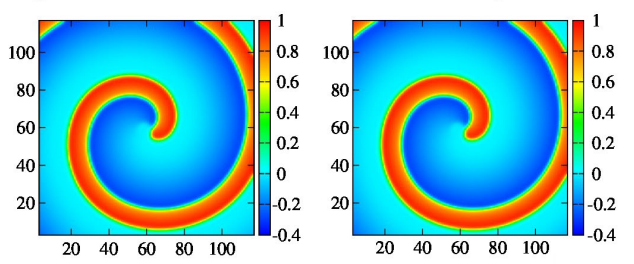

Antiphase locking

System 1

System 2

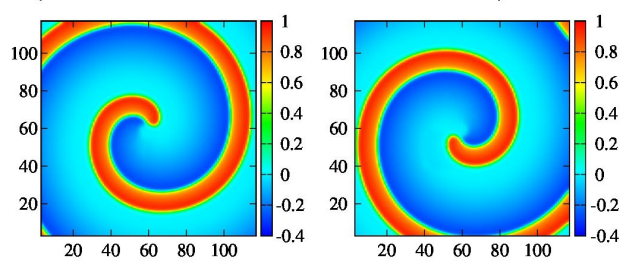

FIG. 4. Rotating spirals. The system is a 2D square of side $L=120$ with no-flux boundary conditions. To pin the spiral at the center, a localized circular heterogeneity of radius $r_{0}=4$ is introduced to the parameter $\alpha(x, y)$ as $\alpha(x, y)=\alpha_{0}+\left(\alpha_{1}-\alpha_{0}\right) \exp \left(-r^{4} / r_{0}^{4}\right)$, where $r=\left[(x-L / 2)^{2}+(y-L / 2)^{2}\right]^{1 / 2}$ is a distance from the center of the system. We assume $\alpha_{0}=0.05$ and $\alpha_{1}=0.5$ so that excitability is the highest at the center. Other parameters are fixed at $\tau^{-1}=0.005, \gamma=2.5, \kappa=0.15$, and $\delta=0$. With these parameters, the oscillation period of the spiral is $T=217.37$. (a) Spiral solution $\mathbf{X}_{0}(x, y ; \theta)=[u(x, y), v(x, y)]$ and the corresponding phase-sensitivity functions $\mathbf{Q}(x, y ; \theta)=\left[Q_{u}(x, y), Q_{v}(x, y)\right]$ at $\theta=0$. (b) Phase-response curves $R(\theta)$ of the spiral normalized by the stimulus intensity $\varepsilon$. The checkerboard-like spatial perturbation is given either to the activator $(u)$ or the inhibitor $(v)$ component, where $s(x, y)=\varepsilon$ for $x, y>L / 2$ or $x, y<L / 2$, and $s(x, y)=0$ otherwise. Results obtained by direct numerical simulations with two different values of $\varepsilon$ are compared with the theory, $R(\theta)=\langle\mathbf{Q}(x, y ; \theta), \mathbf{s}(x, y)\rangle$, where the inner product is taken over the $2 \mathrm{D}$ square $(0 \leq x, y \leq L)$. (c) Evolution of phase differences between two systems coupled through the $u$ component with the coupling intensity matrix $\mathrm{K}=\operatorname{diag}\left(k=2 \times 10^{-4}, 0\right)$, compared with the antisymmetric part of the phase-reduction function $\Gamma_{a}(\phi)$ (rescaled by the coupling intensity $k$ ). Different blue lines show the evolution of $\phi$ for different initial conditions. Both in-phase synchronization (A) and antiphase synchronization (B) can occur, depending on the initial conditions. The solid line corresponds to system 1, and the dashed line corresponds to system 2. (d) Snapshots of the in-phase and antiphase synchronized states.

oscillation is recorded by integrating the original $\mathrm{RD}$ system forward with sufficiently small time grids; then, the adjoint equation is spatially discretized and numerically integrated backward using the recorded time sequence of the limit cycle, with occasional normalization of the solution so that Eq. (4) is satisfied. Owing to the assumed stability of the limit-cycle solution, all modes other than the zero mode corresponding to temporal translational invariance eventually decay (the Floquet theorem), and the resultant solution gives the phase-sensitivity function. 


\section{B. Circulating pulses}

Our first example is a circulating-pulse solution of the FHN model with a wavy tail on a 1D ring of length $L$ [39]. Since the pattern is rigid and the system is translationally symmetric, the phase $\theta$ can simply be identified as the pulse location in this case. Figure 1(a) shows snapshots of the limit-cycle solution $\mathbf{X}_{0}(x ; \theta)=[u(x ; \theta), v(x ; \theta)]$ and the corresponding phase-sensitivity function $\mathbf{Q}(x ; \theta)=$ $\left[Q_{u}(x ; \theta), Q_{v}(x ; \theta)\right]$ for $\theta=0$, both propagating to the right. Results for other values of $\theta$ can simply be obtained by translating Fig. 1(a) in the $x$ direction. An external stimulus applied to the region where $Q_{u, v}(x ; \theta)>0$ will advance the phase of the limit-cycle solution $\mathbf{X}_{0}(x ; \theta)$ (i.e., it pushes the pulse forward to the right), while a stimulus given to the region where $Q_{u, v}(x ; \theta)<0$ will retard the phase of the limit-cycle solution (i.e., it pulls the pulse backward to the left). It is observed that $\mathbf{Q}(x ; \theta)$ is localized near the pulse, indicating that perturbations given only in this region can affect the phase of the pulse. It is also seen that $\mathbf{Q}(x ; \theta)$ has a wavy front, reflecting the wavy tail of the pulse. This counterintuitive result can be explained as follows. The system exhibits localized damped oscillations when it is perturbed at some spatial point. If the pulse propagates into such a region, the pulse location (i.e., the system phase) is either advanced or retarded depending on the timing of the collision, yielding the wavy front of $\mathbf{Q}(x ; \theta)$.

Figure 1(b) compares the PRC $R(\theta)$ of the system to the weak spatial stimulus $\mathbf{s}(x)$ obtained by direct numerical simulations (DNS) with the theoretical results $R(\theta)=\langle\mathbf{Q}(x ; \theta), \mathbf{s}(x)\rangle$, where $\mathbf{Q}(x ; \theta)$ is obtained from the adjoint equation. The stimulus is either a bell shape localized at the center or a cosine curve, and it is given only to the activator component for the sake of simplicity. In each figure, the PRC $R(\theta)$ is normalized by the stimulus intensity $\varepsilon$ as $R(\theta) / \varepsilon$ so that the numerical data fall on the theoretical curve when the linearity assumption Eq. (6) is satisfied [40]. Good agreement is obtained when the intensity $\varepsilon$ of the stimulus is sufficiently small, which confirms the validity of the linearity assumption for a weak stimulus. In the case of the narrowly localized bell-shaped stimulus, the resulting PRC closely resembles $Q_{u}(x ; \theta)$. Indeed, if the stimulus is assumed to be a strictly localized $\delta$ function (though it cannot be realized in real experiments), $R(\theta)$ coincides with $Q_{u}(x ; \theta)$ from Eq. (6). When the stimulus intensity $\varepsilon$ is further increased, nonlinearity in the phase response becomes non-negligible and the present linear theory becomes worse. Eventually, the circulating pulse itself will be destroyed by the stimulus [21]. See Appendix D for more details.

Figure 1(c) shows the synchronization dynamics of the two RD systems, i.e., the evolution of the phase difference $\phi=\theta_{1}-\theta_{2}$ from various initial conditions obtained by the DNS, and the theoretical function $\Gamma_{a}(\phi)$. Reflecting the wavy shapes of $\mathbf{X}_{0}$ and $\mathbf{Q}, \Gamma_{a}(\phi)$ is also wavy with many zeros, which implies the coexistence of multiple stable phase-locking points for the two coupled circulating pulses. This is confirmed by DNS, which shows that the final phase differences are in good agreement with the zero-crossing points of $\Gamma_{a}(\phi)$ with negative $d \Gamma_{a}(\phi) / d \phi$. Figure $1(\mathrm{~d})$ shows several pairs of stably phase-locked pulses (i.e., pairs of pulses stably circulating around the ring with fixed phase differences) obtained by evolving the system from four different initial conditions. The two pulses synchronize where their wavy tails match, yielding multiple stable phase differences as predicted by the phase-reduction analysis. As explained in Sec. II B, when the two pulses are phase locked, their frequency may shift from that of a single unperturbed pulse. See Appendix E for comparison of the numerical data with the theory. Multimodal phase locking similar to Figs. 1(c) and 1(d) is also observed in complex oscillations of delay-differential systems [41].

\section{Oscillating spots}

Our second example is an oscillating spot solution of the 1D FHN system of length $L$ with no-flux boundaries [20]. To pin the spot at the center, the parameter $\alpha$ of the model is assumed to be spatially heterogeneous; namely, the excitability of the system is the largest at the center and the smallest at the boundaries. Note that the pattern is not rigid and the system lacks spatial symmetry. Figure 2(a) shows snapshots of the limit-cycle solution $\mathbf{X}_{0}(x ; \theta)$ and the phase-sensitivity function $\mathbf{Q}(x ; \theta)$ for $\theta=0$. The activator component of $\mathbf{Q}(x ; \theta)$ is sharply localized at both fronts of the spot; namely, the phase $\theta$ of the system is sensitive only to perturbations near the fronts. Figure 2(b) shows $\mathbf{X}_{0}(x ; \theta)$ and corresponding $\mathbf{Q}(x ; \theta)$ for one oscillation period $(0 \leq \theta<2 \pi)$. Perturbations given to the pulse fronts result in an advance or a delay in phase, depending on the timing, i.e., whether the spot is expanding or shrinking. The inhibitor component of $\mathbf{Q}(x ; \theta)$ also reflects the oscillation of the spot. Figure 2(c) shows the PRC $R(\theta)$ to the weak stimulus $\mathbf{S}(x)$, which is either a bell shape or a cosine curve and is only applied to the activator. There is good agreement between the results of numerical simulations and theory. As in the previous case, a stronger stimulus yields nonlinear phase response or leads to collapse of the oscillating spot. See Appendix D for more details.

The synchronization properties of a pair of oscillating spots coupled through the activator component are shown in Fig. 2(d), where the function $\Gamma_{a}(\phi)$ and evolution of the phase difference $\phi$ are plotted. For comparison, two different system sizes, $L=80$ and $L=120$, are used. Since the parameter $\alpha$ is spatially heterogeneous, the shape and oscillation period of the spot vary with $L$. When $L=80$, in-phase synchronization $(\phi=0)$ is linearly stable because $d \Gamma_{a}(\phi=0) / d \phi<0$. In contrast, when $L=120$, in-phase synchronization is unstable and antiphase synchronization $(\phi= \pm \pi)$ becomes stable. This prediction is confirmed by numerical simulations with various initial 
phase differences. Typical snapshots of the synchronized patterns are shown in Fig. 2(e). See Appendix E for the numerical data of the frequency shift, and Videos 1 and 2 for the synchronization dynamics (Appendix F).

\section{Target waves}

As the third example, we consider a target-wave solution $[1,2,18]$ of the 2D FHN model on a square of side $L$ with no-flux boundaries. A circular pacemaker region is created by assuming the parameter $\alpha$ to be heterogeneous. The system is rotationally symmetric around the pacemaker region in this case, but the target pattern is not rigid; the phase should be associated with the temporal dynamics of the pattern.

Figure 3(a) shows the limit-cycle solution $\mathbf{X}_{0}(x, y ; \theta)$ and the corresponding phase-sensitivity function $\mathbf{Q}(x, y ; \theta)$ for $\theta=0$. As $\theta$ increases, $\mathbf{X}_{0}(x, y ; \theta)$ undergoes oscillations corresponding to the emission of concentric target waves from the pacemaker, and $\mathbf{Q}(x, y ; \theta)$ oscillates accordingly (see Appendix C). Reflecting the fact that the pacemaker dominates overall rhythms of the system, $\mathbf{Q}(x, y ; \theta)$ is localized at the pacemaker. Figure 3(b) shows the PRC $R(\theta)$ obtained by applying a weak cosine spatial stimulus $\mathbf{s}(x, y)$ to either the activator or the inhibitor component. The numerical results are in good agreement with the theory. See Appendix D for nonlinear PRCs to stronger stimuli.

Figure 3(c) shows synchronization between two target waves. Here, we consider counterpropagating target waves; i.e., one of the RD systems is inverted in the $x$ direction, as shown in Fig. 3(e). The function $\Gamma_{a}(\phi)$ has five zeros, with the in-phase $(\phi=0)$ and antiphase $(\phi= \pm \pi)$ synchronized states both being stable. Therefore, depending on the initial conditions, the two target waves can exhibit both types of synchronization, as confirmed by numerical simulations. Figure 3(d) shows time sequences of the activator at the center of the two systems corresponding to the in-phase and antiphase synchronized states, and Fig. 3(e) shows the corresponding snapshots. See Appendix E for the numerical data of the frequency shift, and Videos 3 and 4 for the synchronization dynamics (Appendix F).

\section{E. Rotating spirals}

Our final example is a rotating-spiral solution $[1,2,17,18,26]$ of the FHN model on a 2D square of side $L$ with no-flux boundaries. Synchronization between a pair of rotating spirals was experimentally studied in Ref. [26]. Here, to pin the core of the spiral at the center of the system, circular heterogeneity in the parameter $\alpha$ is introduced. The spiral rigidly rotates around this pinning region without changing its shape.

Figure 4(a) shows snapshots of the spiral solution $\mathbf{X}_{0}(x, y ; \theta)$ and the corresponding phase-sensitivity function $\mathbf{Q}(x, y ; \theta)$ at $\theta=0$. Both rotate in the clockwise direction as $\theta$ increases. Since the system is symmetric with respect to spatial rotation around the center and since the pattern is rigid, the phase $\theta$ simply corresponds to the rotation angle, and the results for other values of $\theta$ can be obtained by rotating Fig. 4(a). As in the other cases, $\mathbf{Q}(x, y ; \theta)$ is strongly localized near the core of the spiral, indicating that the spiral tip dominates the overall phase of the system; perturbations given only to this region can affect the overall system phase. Figure 4(b) compares the PRCs obtained by DNS with the theory, $R(\theta)=$ $\langle\mathbf{Q}(x, y ; \theta), \mathbf{s}(x, y)\rangle$, to a checkerboard-like stimulus $s(x, y)$ applied either to the activator or the inhibitor, showing good agreement. See Appendix D for the nonlinear phase response to stronger stimuli.

Figure 4(c) shows the synchronization process between two spirals. As expected from the function $\Gamma_{a}(\phi)$ with five zeros, the two spirals can exhibit either in-phase $(\phi=0)$ or antiphase $(\phi= \pm \pi)$ synchronization, as determined by the initial conditions. Typical time sequences of the activator component measured at $x=L / 4, y=L / 2$ in the in-phase and antiphase synchronized states are shown in Fig. 4(d), and typical snapshots of the synchronized spirals are shown in Fig. 4(e). See Appendix E for the numerical data of the frequency shift and Videos 5 and 6 for the synchronization dynamics (Appendix F).

\section{CONCLUDING REMARKS}

We developed a phase-reduction theory for limit-cycle solutions of infinite-dimensional RD systems and illustrated its validity by analyzing mutual synchronization of a pair of RD systems exhibiting rhythmic dynamics. Our theory does not assume rigidity and spatial symmetry; therefore, it is generally applicable to a wide class of rhythmic spatiotemporal dynamics in RD systems and will be a basis for controlling and designing spatiotemporal rhythms in various RD systems. Though we analyzed only a pair of coupled RD systems, the theory can readily be applied, for example, to the analysis of phase locking to periodic external stimuli and noise-induced synchronization [1-3,5,6,11-13,42-44] of spatiotemporal rhythms.

As assumed in Sec. II, the frequency of the pattern should be constant over the space, and the whole system should maintain its consistency even if disturbed by perturbations. Under this condition, the present theory can also treat the effect of internal coupling within the system, e.g., nonlocal feedback coupling among distant regions of the same RD medium. When the perturbation comes from the RD system itself, the system would be described by $\partial \mathbf{X}(\mathbf{r}, t) / \partial t=\mathbf{F}(\mathbf{X}, \mathbf{r})+D \nabla^{2} \mathbf{X}+$ $\mathbf{p}\left\{\mathbf{X}\left(\mathbf{r}, t-t_{0}\right)\right\}$, where $\mathbf{p}\left\{\mathbf{X}\left(\mathbf{r}, t-t_{0}\right)\right\}$ represents some weak feedback functional of the system state $\mathbf{X}\left(\mathbf{r}, t-t_{0}\right)$ at time $t-t_{0}\left(t_{0} \geq 0\right.$ is a delay time $)$. Then, using the phase-sensitivity function $\mathbf{Q}(\mathbf{r} ; \theta)$, the lowest-order approximate phase equation can be written down as $\dot{\theta}(t)=\omega+\left\langle\mathbf{Q}(\mathbf{r} ; \theta(t)), \mathbf{p}\left\{\mathbf{X}_{0}\left[\mathbf{r} ; \theta\left(t-t_{0}\right)\right]\right\}\right\rangle$. Note that the feedback term should be sufficiently small so that 
the system does not split into multiple regions with different frequencies.

In Figs. 1-4, we notice that the phase-sensitivity functions $\mathbf{Q}(\mathbf{r} ; \theta)$ of the rhythmic patterns are localized in space. Such localization tends to occur near dynamically important structures of each pattern, i.e., near the wavefronts of the circulating pulse and oscillating spot (Figs. 1 and 2), and around the cores of the target wave and rotating spiral (Figs. 3 and 4). This is physically plausible because these regions control the overall dynamics of the whole patterns. In this sense, $\mathbf{Q}(\mathbf{r} ; \theta)$ characterizes dynamically important regions of the system. Of course, for other rhythmic patterns without such structures, e.g., for smooth nonlinear waves in spatially uniform oscillatory media, localization of the phase-sensitivity function may not be apparent.

As we explained in Sec. I, reduction of RD systems exhibiting rigidly traveling pulses and rotating spirals to low-dimensional phase equations has been considered in many studies $[2,24,25,28-36]$. They can be considered phase-reduction theories for RD systems from our present viewpoint, though the definition of the "phase" as the oscillation phase may not be explicit. As we stressed, the merit of the present study is that it clearly gives a systematic phase-reduction theory applicable to general reactiondiffusion systems exhibiting limit-cycle oscillations without assuming any spatial symmetry or rigidity.

Phase-reduction theories for infinite-dimensional dynamical systems undergoing stable periodic oscillations have also been developed in several other contexts. For example, we derived macroscopic phase equations for systems of globally coupled oscillators and excitable units exhibiting collective rhythms in our recent works $[45,46]$. The theory can be considered a phase-reduction theory for a nonlinear Fokker-Planck equation describing the one-body probability density of the dynamical units in the continuum limit. Recently, we have also succeeded in deriving phase equations for steadily oscillating convection in a HeleShaw cell $[47,48]$ described by fluid equations from the viewpoint of phase-reduction theory. Also, the phasereduction theory for delay-induced oscillations recently developed by Novičenko and Pyragas [49] and by us [41] is also for infinite-dimensional dynamical systems because delay-differential equations have infinite-dimensional state space. In this case, an appropriate inner product (bilinear form) for delay-differential equations, which is different from the $L_{2}$ norm used for the partial-differential equations, should be used. These studies imply that it is generally possible to extend the phase-reduction theory to various infinite-dimensional dynamical systems undergoing limitcycle oscillations, which will give us a simple unified way to analyze and control their rhythmic dynamics.

In the present study, we consider only the case in which the RD system exhibits a stable limit-cycle oscillation with a single phase variable. Generally, a reaction-diffusion system can also possess other phase variables associated with other neutrally stable directions. For example, rigidly rotating spirals without a pinning force in 2D extended media generally have three phases, i.e., one rotation phase and two spatial phases (the $x y$ coordinates of the core). If the spiral core meanders along a closed circular trajectory, the system possesses a stable 2-torus with two phase variables. Phase equations for such spirals have been derived in several studies [35,36] under the rigidity assumption of the spiral patterns. Because phase-reduction theories for quasiperiodic oscillations have already been developed [50,51,55] for low-dimensional limit-torus oscillators, we will be able to develop similar theories for infinite-dimensional dynamical systems exhibiting limit-torus oscillations without assuming rigidity or spatial symmetry. As an attempt in this direction, we recently analyzed oscillatory Hele-Shaw convection with translational symmetry, which possesses two phase variables [52]. It should also be possible to develop a more general phasereduction theory that is applicable to RD systems possessing stable limit-torus solutions.

\section{ACKNOWLEDGMENTS}

We thank Dr. I. Z. Kiss and Dr. R. Toenjes for useful comments. H. N. acknowledges financial support from JSPS KAKENHI Grants No. 25540108, No. 26103510 , and No. 26120513, the CREST Kokubu project of JST, and the FIRST Aihara project of JSPS. T. Y. acknowledges financial support from JSPS KAKENHI Grant No. 24540417. Y. K. acknowledges financial support from JSPS KAKENHI Grant No. 25800222.

\section{APPENDIX A: PHASE REDUCTION OF ORDINARY LIMIT-CYCLE OSCILLATORS}

In this appendix, we review the classical phase-reduction theory for ordinary limit-cycle oscillators described by finite-dimensional ODEs. See Refs. [1-5] for details of the theory and applications to various synchronization phenomena in systems of coupled oscillators.

\section{Geometric formulation of the phase-reduction theory}

We consider a limit-cycle oscillator described by the ODE

$$
\dot{\mathbf{X}}(t)=\mathbf{F}[\mathbf{X}(t)],
$$

where $\mathbf{X}(t)$ is a $d \geq 2$-dimensional vector representing the oscillator state at time $t$ and $\mathbf{F}$ determines its dynamics. Suppose that Eq. (A1) has a stable limit-cycle solution of period $T$,

$$
\chi: \mathbf{X}_{0}(t)=\mathbf{X}_{0}(t+T),
$$

which is denoted as $\chi$. We introduce a phase $\theta(t) \in[0,2 \pi)$ to the state $\mathbf{X}_{0}(t)$ on $\chi$ in such a way that $\theta(t)$ increases with 
a constant frequency $\omega=2 \pi / T$ as $\mathbf{X}_{0}(t)$ evolves along $\chi$ under Eq. (A1). This can be performed by choosing a certain state $\mathbf{X}_{0}(t=0)$ on $\chi$ as the origin of the phase, i.e., $\theta=0$, and assigning a phase value

$$
\theta=\omega t(\bmod 2 \pi)
$$

to the oscillator state $\mathbf{X}_{0}(t)$ on $\chi(t \geq 0)$ evolving under Eq. (A1) from the phase origin $\mathbf{X}_{0}(t=0)$. Namely, we identify the oscillator phase with the time multiplied by the frequency. We will denote the oscillator state on $\chi$ with the phase value $\theta$ as $\mathbf{X}_{0}(\theta)$ henceforth.

The above definition of the phase on $\chi$ can be extended to the whole basin of $\chi$ by assigning the same phase value $\theta(t)$ to the set of oscillator states $\{\mathbf{X}(t)\}$ that asymptotically approach the oscillator state $\mathbf{X}_{0}[\theta(t)]$ on $\chi$ under Eq. (A1), i.e.,

$$
\lim _{t \rightarrow+\infty}\left|\mathbf{X}(t)-\mathbf{X}_{0}[\theta(t)]\right|=0
$$

where $|\cdots|$ represents the ordinary vector norm. This defines a phase function

$$
\theta=\Theta(\mathbf{X}) \in[0,2 \pi)
$$

that maps a given oscillator state $\mathbf{X}$ in the basin of $\chi$ to a scalar phase $\theta$. It is clear that the phase $\theta(t)=\Theta[\mathbf{X}(t)]$ of the state $\mathbf{X}(t)$ evolving under Eq. (A1) obeys a simple phase equation,

$$
\dot{\theta}(t)=\omega,
$$

not only on the limit-cycle solution $\chi$ but also in the whole basin of $\chi$. Using the chain rule for the derivatives, it can be shown that

(a)

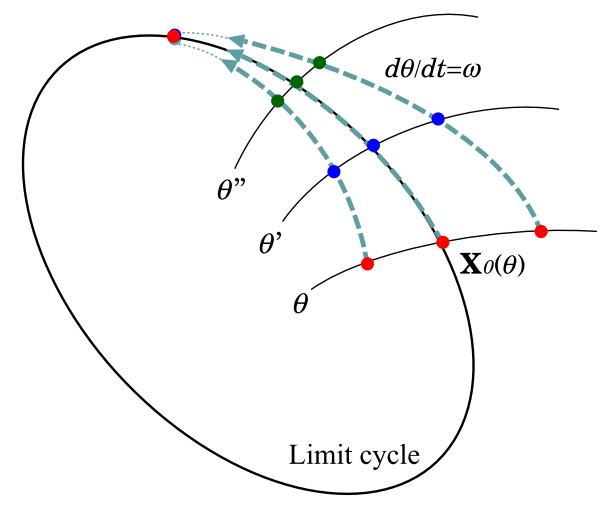

$$
\begin{aligned}
\dot{\theta}(t) & =\frac{d}{d t} \Theta[\mathbf{X}(t)]=\left.\frac{\partial \Theta(\mathbf{X})}{\partial \mathbf{X}}\right|_{\mathbf{X}=\mathbf{X}(t)} \cdot \frac{d \mathbf{X}(t)}{d t} \\
& =\left.\frac{\partial \Theta(\mathbf{X})}{\partial \mathbf{X}}\right|_{\mathbf{X}=\mathbf{X}(t)} \cdot \mathbf{F}[\mathbf{X}(t)]=\omega,
\end{aligned}
$$

where $\partial \Theta(\mathbf{X}) /\left.\partial \mathbf{X}\right|_{\mathbf{X}=\mathbf{X}(t)}$ is the gradient of the phase function $\Theta(\mathbf{X})$ at $\mathbf{X}=\mathbf{X}(t)$. Thus, the phase function $\Theta(\mathbf{X})$ should satisfy

$$
\frac{\partial \Theta(\mathbf{X})}{\partial \mathbf{X}} \cdot \mathbf{F}(\mathbf{X})=\omega
$$

in the basin of $\chi$. The set of oscillator states sharing the same phase value is called the isochron and is the fundamental concept in the analysis of limit-cycle oscillators $[1-5,37,38]$. The whole basin of $\chi$ is foliated by such isochrons. See Fig. 5(a) for a schematic illustration of the isochrons.

Now we consider the case in which the limit-cycle oscillator is weakly perturbed as

$$
\dot{\mathbf{X}}(t)=\mathbf{F}[\mathbf{X}(t)]+\mathbf{p}[\mathbf{X}(t), t]
$$

where the perturbation $\mathbf{p}(\mathbf{X}, t)$, generally a function of the oscillator state $\mathbf{X}$ and time $t$, is assumed to be sufficiently weak so that the original limit-cycle solution $\chi$ is only slightly deformed. From Eq. (A7), the phase $\theta(t)=$ $\Theta[\mathbf{X}(t)]$ of the perturbed oscillator obeys

$$
\begin{aligned}
\dot{\theta}(t) & =\left.\frac{\partial \Theta(\mathbf{X})}{\partial \mathbf{X}}\right|_{\mathbf{X}=\mathbf{X}(t)} \cdot\{\mathbf{F}[\mathbf{X}(t)]+\mathbf{p}[\mathbf{X}(t), t]\} \\
& =\omega+\left.\frac{\partial \Theta(\mathbf{X})}{\partial \mathbf{X}}\right|_{\mathbf{X}=\mathbf{X}(t)} \cdot \mathbf{p}[\mathbf{X}(t), t] .
\end{aligned}
$$

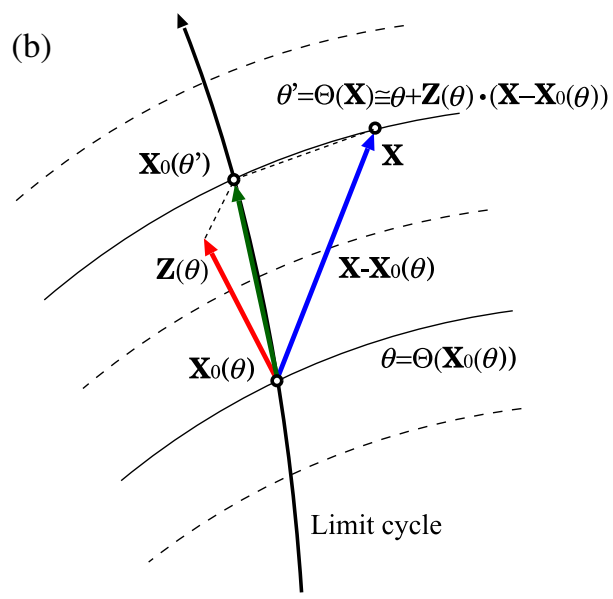

FIG. 5. (a) Isochrons of a limit cycle. The same phase value is assigned to the oscillator states that asymptotically converge to the same state on the limit cycle. (b) Linear approximation of the phase function near the limit cycle. 
However, this is not a closed equation for $\theta(t)$ because the gradient $\partial \Theta(\mathbf{X}) /\left.\partial \mathbf{X}\right|_{\mathbf{X}=\mathbf{X}(t)}$ and the perturbation $\mathbf{p}[\mathbf{X}(t), t]$ still depend on $\mathbf{X}(t)$. To obtain a closed equation for $\theta(t)$, $\mathbf{X}(t)$ in these terms is replaced by $\mathbf{X}_{0}[\theta(t)]$ at the lowestorder approximation, assuming that the perturbation $\mathbf{p}[\mathbf{X}(t), t]$ is sufficiently weak so that $\mathbf{X}(t)$ does not significantly deviate from $\mathbf{X}_{0}[\theta(t)]$ on $\chi$. This yields an approximate, closed phase equation for $\theta(t)$,

$$
\dot{\theta}(t) \simeq \omega+\left.\frac{\partial \Theta(\mathbf{X})}{\partial \mathbf{X}}\right|_{\mathbf{X}=\mathbf{X}_{0}(\theta(t))} \cdot \mathbf{p}\left(\mathbf{X}_{0}[\theta(t)], t\right),
$$

which is correct up to $O(|\mathbf{p}|)$. Thus, by denoting $\mathbf{p}(\theta, t)=$ $\mathbf{p}\left[\mathbf{X}_{0}(\theta), t\right]$ and introducing a function

$$
\mathbf{Z}(\theta)=\left.\frac{\partial \Theta(\mathbf{X})}{\partial \mathbf{X}}\right|_{\mathbf{X}=\mathbf{x}_{0}(\theta)},
$$

the $d$-dimensional ODE (A9) describing a perturbed limit cycle can be reduced to a simple one-dimensional phase equation

$$
\dot{\theta}(t)=\omega+\mathbf{Z}[\theta(t)] \cdot \mathbf{p}[\theta(t), t]
$$

at the lowest order in the perturbation.

The key quantity for this approximation is the phasesensitivity function $\mathbf{Z}(\theta)$ defined in Eq. (A12), which is the gradient of the isochron estimated at $\mathbf{X}=\mathbf{X}_{0}(\theta)$ on the limit-cycle solution $\chi$. The function $\mathbf{Z}(\theta)$ quantifies the linear phase-response property of the oscillator state $\mathbf{X}_{0}(\theta)$ with the phase $\theta$ on $\chi$ to infinitesimal perturbations. If $\mathbf{X}_{0}(\theta)$ is instantaneously perturbed by a weak stimulus $\mathbf{s}$, the resulting phase response is given by

$$
R(\theta)=\mathbf{Z}(\theta) \cdot \mathbf{s}
$$

under a linear approximation. This $R(\theta)$ is called the phaseresponse curve (PRC) of the limit-cycle oscillator described by Eq. (A1). The PRC can be obtained by applying impulsive perturbations to a limit-cycle oscillator and has been measured in various experimental systems [1].

\section{Linear theory around the limit-cycle solution}

Though we have developed a formal geometric theory by assuming the existence of the phase function $\Theta(\mathbf{X})$, it is generally impossible to obtain $\Theta(\mathbf{X})$ explicitly, except for a few simple models of limit-cycle oscillators. However, to obtain the lowest-order phase equation (A12) for weak perturbations, only the phase-sensitivity function $\mathbf{Z}(\theta)$ is actually necessary. As we show below, the function $\mathbf{Z}(\theta)$ can be obtained as the $2 \pi$-periodic solution to the following adjoint equation $[3,5]$ :

$$
\omega \frac{d \mathbf{Z}(\theta)}{d \theta}=-\mathrm{J}(\theta)^{\dagger} \mathbf{Z}(\theta),
$$

with the constraint $\mathbf{Z}(\theta) \cdot \mathbf{F}\left[\mathbf{X}_{0}(\theta)\right]=\omega$, or equivalently,

$$
\mathbf{Z}(\theta) \cdot \frac{d}{d \theta} \mathbf{X}_{0}(\theta)=1,
$$

for $0 \leq \theta<2 \pi$, where $\mathbf{J}(\theta)=\mathbf{J}\left[\mathbf{X}_{0}(\theta)\right]$ is the Jacobi matrix of $\mathbf{F}(\mathbf{X})$ at $\mathbf{X}=\mathbf{X}_{0}(\theta)$ on $\chi$ and ${ }^{\dagger}$ indicates the matrix transpose.

The adjoint equation (A15) and the normalization condition (A16) can be derived in several different ways. Here, we use a simple argument by Brown, Moehlis, and Holmes [4] with an emphasis on the linear approximation of the isochrons near the limit cycle [see Eq. (A18) below]. We use the same idea to develop a phase-reduction theory for the limit-cycle solutions of reaction-diffusion systems in Appendix B. See Fig. 5(b) for a schematic illustration.

We first note that, when $\left|\mathbf{X}-\mathbf{X}_{0}(\theta)\right|$ is sufficiently small, the phase function $\Theta(\mathbf{X})$ for the ODE can be expanded in a Taylor series around $\mathbf{X}_{0}(\theta)$ as

$$
\begin{aligned}
\Theta(\mathbf{X})= & \Theta\left[\mathbf{X}_{0}(\theta)+\mathbf{X}-\mathbf{X}_{0}(\theta)\right] \\
= & \Theta\left[\mathbf{X}_{0}(\theta)\right]+\left.\frac{\partial \Theta(\mathbf{X})}{\partial \mathbf{X}}\right|_{\mathbf{X}=\mathbf{X}_{0}(\theta)} \cdot\left[\mathbf{X}-\mathbf{X}_{0}(\theta)\right] \\
& +O\left[\left|\mathbf{X}-\mathbf{X}_{0}(\theta)\right|^{2}\right] \\
= & \theta+\mathbf{Z}(\theta) \cdot\left[\mathbf{X}-\mathbf{X}_{0}(\theta)\right]+O\left[\left|\mathbf{X}-\mathbf{X}_{0}(\theta)\right|^{2}\right]
\end{aligned}
$$

using the phase-sensitivity function $\mathbf{Z}(\theta)$ in Eq. (A12). Therefore, if the oscillator state $\mathbf{X}$ is close to the oscillator state $\mathbf{X}_{0}(\theta)$ with the phase $\theta$ on the limit-cycle solution $\chi$, $\Theta(\mathbf{X})$ can be linearly approximated as

$$
\Theta(\mathbf{X}) \simeq \theta+\mathbf{Z}(\theta) \cdot\left[\mathbf{X}-\mathbf{X}_{0}(\theta)\right]
$$

Suppose we have an initial state $\mathbf{X}_{0}(t=0)=\mathbf{X}(\theta=0)$ on $\chi$, and a slightly perturbed initial state

$$
\mathbf{X}(t=0)=\mathbf{X}_{0}(\theta=0)+\mathbf{y}(t=0)
$$

near $\mathbf{X}_{0}(\theta=0)$, where $\mathbf{y}(t=0)$ is a small perturbation given to $\mathbf{X}_{0}(\theta=0)$. We evolve these two states without applying further perturbations. Then, from Eq. (A1), the linearized equation for the small perturbation $\mathbf{y}(t)=$ $\mathbf{X}(t)-\mathbf{X}_{0}[\theta(t)]$ is given by

$$
\frac{d}{d t} \mathbf{y}(t)=\mathbf{J}[\theta(t)] \mathbf{y}(t),
$$

where $\mathbf{J}[\theta(t)]=\mathbf{J}\left(\mathbf{X}_{0}[\theta(t)]\right)=\mathbf{D F}\left(\mathbf{X}_{0}[\theta(t)]\right)$ is the Jacobi matrix of $\mathbf{F}(\mathbf{X})$ at $\mathbf{X}=\mathbf{X}_{0}(\theta)$ on $\chi$. From Eq. (A18), the phase of the unperturbed state is given by $\theta(t)=$ $\Theta\left(\mathbf{X}_{0}[\theta(t)]\right)=\omega t$ and that of the perturbed state can be expressed as 


$$
\begin{aligned}
\theta^{\prime}(t) & =\Theta[\mathbf{X}(t)] \\
& =\Theta\left(\mathbf{X}_{0}[\theta(t)]+\mathbf{y}(t)\right) \simeq \theta(t)+\mathbf{Z}[\theta(t)] \cdot \mathbf{y}(t)
\end{aligned}
$$

under the linear approximation. Note here that $\theta^{\prime}(t)$ should also increase with a constant frequency $\omega$, i.e., $d \theta^{\prime}(t) / d t=\omega$ within the linear approximation, because no perturbation is given after $t=0$. Thus, the following equation should hold for $\mathbf{y}(t)$ evolving from arbitrary $\mathbf{y}(t=0)$ :

$$
\begin{aligned}
0 & =\frac{d}{d t}\left\{\theta^{\prime}(t)-\theta(t)\right\}=\frac{d}{d t}\{\mathbf{Z}[\theta(t)] \cdot \mathbf{y}(t)\} \\
& =\frac{d \mathbf{Z}[\theta(t)]}{d t} \cdot \mathbf{y}(t)+\mathbf{Z}[\theta(t)] \cdot \frac{d \mathbf{y}(t)}{d t} \\
& =\frac{d \mathbf{Z}[\theta(t)]}{d t} \cdot \mathbf{y}(t)+\mathbf{Z}[\theta(t)] \cdot \mathbf{J}[\theta(t)] \mathbf{y}(t) \\
& =\left\{\frac{d}{d t} \mathbf{Z}[\theta(t)]+\mathbf{J}[\theta(t)]^{\dagger} \mathbf{Z}[\theta(t)]\right\} \cdot \mathbf{y}(t) .
\end{aligned}
$$

Therefore, $\mathbf{Z}[\theta(t)]$ should satisfy the following adjoint equation:

$$
\frac{d}{d t} \mathbf{Z}[\theta(t)]=-\mathbf{J}[\theta(t)]^{\dagger} \mathbf{Z}(\theta(t)),
$$

which is equivalent to Eq. (A15) by the relation $d / d t=$ $\omega d / d \theta$ (note that $\theta=\omega t$ ). To obtain the normalization condition Eq. (A16), we differentiate the identity $\theta(t)=$ $\Theta\left(\mathbf{X}_{0}[\theta(t)]\right)=\omega t$ by $t$, which yields

$$
\begin{aligned}
\omega & =\frac{d}{d t} \theta(t)=\left.\frac{\partial \Theta(\mathbf{X})}{\partial \mathbf{X}}\right|_{\mathbf{X}=\mathbf{X}_{0}[\theta(t)]} \cdot \frac{d}{d t} \mathbf{X}_{0}[\theta(t)] \\
& =\mathbf{Z}[\theta(t)] \cdot \mathbf{F}\left(\mathbf{X}_{0}[\theta(t)]\right) .
\end{aligned}
$$

This gives the normalization condition Eq. (A16), again by the relation $d / d t=\omega d / d \theta$.

Thus, the function $\mathbf{Z}(\theta)$ can be obtained by solving the adjoint equation (A15) under the normalization condition Eq. (A16), and the phase function near $\chi$ is given by Eq. (A18) within a linear approximation. It can also be shown that $\mathbf{Z}(\theta)$ is the unique solution to Eq. (A15) by using the Floquet theorem characterizing the linear stability of the limit cycle, since $\mathbf{Z}(\theta)$ is essentially the Floquet eigenvector with the zero Floquet exponent [2-5]. In actual numerical calculations, it is useful to integrate Eq. (A15) backward in time to avoid numerical overflow, with occasional normalization using Eq. (A16) [5]. Then, by virtue of the Floquet theorem, only the functional component corresponding to $\mathbf{Z}(\theta)$ remains.

Once we obtain the frequency $\omega$ and the phasesensitivity function $\mathbf{Z}(\theta)$, we can write down the approximate phase equation (A13) for a weakly perturbed limit-cycle oscillator described by Eq. (A9). This approximation, called the phase reduction, greatly simplifies theoretical analysis of weakly perturbed limit cycles and has been extensively used for analyzing synchronization dynamics of weakly interacting nonlinear oscillators [1-5].

\section{APPENDIX B: DERIVATION OF THE PHASE-REDUCTION THEORY FOR REACTION-DIFFUSION SYSTEMS}

In this section, we give a full derivation of the phase-reduction theory for RD systems that we briefly summarized in Sec. II A. Our aim is to derive a simple onedimensional phase equation for rhythmic spatiotemporal patterns described as limit-cycle solutions of RD systems without recourse to spatial symmetry of the patterns. We do not require the patterns to be rigidly translating or rotating in the RD medium without changing their spatial profiles, as typically assumed in the conventional derivation of the phase equations for RD systems. Such rhythmic patterns can thus include oscillating spots and target waves, which vary their spatial profiles periodically. Rigidly circulating waves or rotating spirals with spatial translational or rotational symmetry are also limit-cycle solutions of RD systems, and thus they can also be treated in the same framework as we showed in the previous section.

Our strategy is to generalize the conventional phasereduction theory for ordinary limit cycles described by ODEs (see Appendix A for comparison), which assumes only temporal translational symmetry of the oscillator dynamics, to limit-cycle solutions of infinite-dimensional $\mathrm{RD}$ systems, thereby avoiding the assumptions on spatial symmetry. We can develop the theory almost in parallel with the ODE case by noticing that the finite-dimensional vector $\mathbf{X}(t)$ is replaced by a vector field $\mathbf{X}(\mathbf{r}, t)$, and correspondingly, the ordinary dot product of two vectors is replaced by the inner product of two vector fields.

\section{Geometric formulation of the phase-reduction theory}

We consider a RD equation of the form

$$
\frac{\partial}{\partial t} \mathbf{X}(\mathbf{r}, t)=\mathbf{F}[\mathbf{X}(\mathbf{r}, t), \mathbf{r}]+\mathrm{D} \nabla^{2} \mathbf{X}(\mathbf{r}, t),
$$

where the $d$-dimensional vector $\mathbf{X}(\mathbf{r}, t)$ represents the state of the RD medium at point $\mathbf{r}$ in the $n$-dimensional space at time $t, \mathbf{F}(\mathbf{X}, \mathbf{r})$ specifies local reaction dynamics at point $\mathbf{r}$, and $\mathrm{D} \nabla^{2} \mathbf{X}(\mathbf{r}, t)$ represents diffusion of $\mathbf{X}$ over the medium with a constant diffusion matrix D. Explicit dependence of $\mathbf{F}$ on $\mathbf{r}$, such as heterogeneity of the medium, may exist. Appropriate boundary conditions (e.g., periodic or no-flux) for the problem under consideration are introduced. We assume that Eq. (B1) has a stable limit-cycle solution of period $T$,

$$
\chi: \mathbf{X}_{0}(\mathbf{r}, t)=\mathbf{X}_{0}(\mathbf{r}, t+T),
$$

which is denoted by $\chi$. As in the ODE case, we first define a phase $\theta(t) \in[0,2 \pi)$ of the system state $\mathbf{X}_{0}(\mathbf{r}, t)$ on the 
limit-cycle solution $\chi$ so that $\theta(t)$ increases with a constant frequency $\omega=2 \pi / T$ as $\mathbf{X}_{0}(\mathbf{r}, t)$ evolves on $\chi$ under Eq. (B1). This is performed by identifying the phase with the time multiplied by the frequency. Namely, we choose a certain system state $\mathbf{X}_{0}(\mathbf{r}, t=0)$ on $\chi$ as the origin of the phase, $\theta=0$, and assign a phase value

$$
\theta=\omega t(\bmod 2 \pi)
$$

to a state $\mathbf{X}_{0}(\mathbf{r}, t)$ on $\chi(t \geq 0)$ evolving under Eq. (B1) from the phase origin $\mathbf{X}_{0}(\mathbf{r}, t=0)$. We will denote the system state on $\chi$ with the phase value $\theta$ as $\mathbf{X}_{0}(\mathbf{r} ; \theta)$ henceforth.

Next, we need to extend the definition of the phase to the basin of $\chi$. As in the ODE case, we assign the same phase value $\theta(t)$ to the set of system states $\{\mathbf{X}(\mathbf{r}, t)\}$ that eventually converge to the system state $\mathbf{X}_{0}[\mathbf{r} ; \theta(t)]$ on $\chi$ under Eq. (B1), namely,

$$
\lim _{t \rightarrow+\infty}\left\|\mathbf{X}(\mathbf{r}, t)-\mathbf{X}_{0}[\mathbf{r} ; \theta(t)]\right\|=0 .
$$

Here, $\|\cdots\|$ denotes the $L^{2}$ norm of a spatial pattern defined as $\|\mathbf{A}(\mathbf{r})\|^{2}=\langle\mathbf{A}(\mathbf{r}), \mathbf{A}(\mathbf{r})\rangle$, and the inner product between two spatial patterns $\mathbf{A}(\mathbf{r})$ and $\mathbf{B}(\mathbf{r})$ is defined as

$$
\langle\mathbf{A}(\mathbf{r}), \mathbf{B}(\mathbf{r})\rangle=\int \mathbf{A}(\mathbf{r}) \cdot \mathbf{B}(\mathbf{r}) d \mathbf{r} .
$$

The integral is taken over the considered spatial domain with appropriate boundary conditions. This introduces a phase functional

$$
\theta=\Theta\{\mathbf{X}(\mathbf{r})\} \in[0,2 \pi)
$$

that maps a given system state $\mathbf{X}(\mathbf{r})$ in the basin of $\chi$ to a scalar phase $\theta$. Then, the phase $\theta(t)=\Theta\{\mathbf{X}(\mathbf{r}, t)\}$ of the state $\mathbf{X}(\mathbf{r}, t)$ evolving under Eq. (B1) will constantly obey

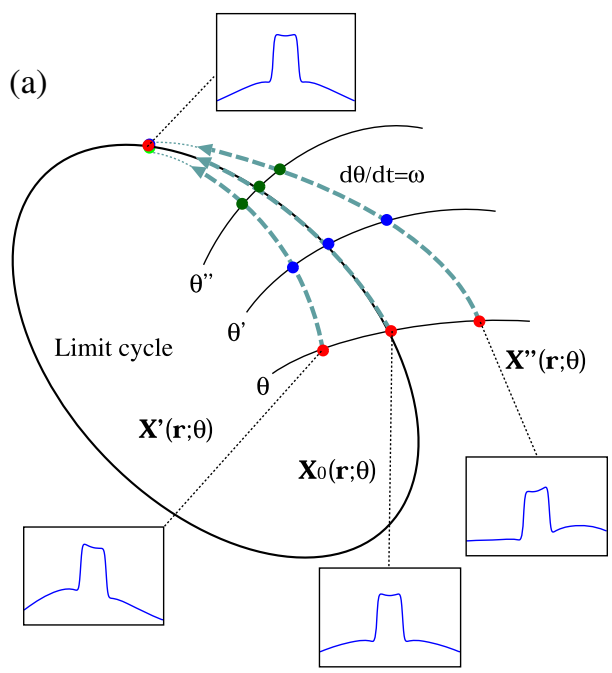

$$
\dot{\theta}(t)=\omega
$$

not only on the limit-cycle solution $\chi$ but also in the whole basin of $\chi$. Using the chain rule for the functional derivatives, the above equation can be written as

$$
\begin{aligned}
\dot{\theta}(t) & =\frac{d}{d t} \Theta\{\mathbf{X}(\mathbf{r}, t)\}=\left\langle\left.\frac{\delta \Theta\{\mathbf{X}(\mathbf{r})\}}{\delta \mathbf{X}(\mathbf{r})}\right|_{\mathbf{X}(\mathbf{r})=\mathbf{X}(\mathbf{r}, t)}, \frac{\partial}{\partial t} \mathbf{X}(\mathbf{r}, t)\right\rangle \\
& =\left\langle\left.\frac{\delta \Theta\{\mathbf{X}(\mathbf{r})\}}{\delta \mathbf{X}(\mathbf{r})}\right|_{\mathbf{X}(\mathbf{r})=\mathbf{X}(\mathbf{r}, t)}, \mathbf{F}[\mathbf{X}(\mathbf{r}, t), \mathbf{r}]+\mathrm{D} \nabla^{2} \mathbf{X}(\mathbf{r}, t)\right\rangle \\
& =\omega,
\end{aligned}
$$

where $\delta \Theta\{\mathbf{X}(\mathbf{r})\} / \delta \mathbf{X}(\mathbf{r})$ is the functional derivative of $\Theta\{\mathbf{X}(\mathbf{r})\}$ with respect to $\mathbf{X}(\mathbf{r})=\mathbf{X}(\mathbf{r}, t)$. Thus, the phase functional $\Theta\{\mathbf{X}(\mathbf{r})\}$ should satisfy

$$
\left\langle\frac{\delta \Theta\{\mathbf{X}(\mathbf{r})\}}{\delta \mathbf{X}(\mathbf{r})}, \mathbf{F}[\mathbf{X}(\mathbf{r}), \mathbf{r}]+\mathrm{D} \nabla^{2} \mathbf{X}(\mathbf{r})\right\rangle=\omega
$$

in the basin of $\chi$. We call a set of system states sharing the same phase value the isochron of the RD system, generalizing the same notion for ODEs (Appendix A). The whole basin of $\chi$ is foliated by such isochrons [53]. See Fig. 6(a) for a schematic illustration.

Now we consider the case in which the RD system is weakly perturbed as

$\frac{\partial}{\partial t} \mathbf{X}(\mathbf{r}, t)=\mathbf{F}[\mathbf{X}(\mathbf{r}, t), \mathbf{r}]+\mathrm{D}^{2} \mathbf{X}(\mathbf{r}, t)+\mathbf{p}\{\mathbf{X}(\mathbf{r}, t), \mathbf{r}, t\}$,

where the perturbation $\mathbf{p}\{\mathbf{X}(\mathbf{r}, t), \mathbf{r}, t\}$ is generally a functional of the state $\mathbf{X}(\mathbf{r}, t)$, location $\mathbf{r}$, and time $t$. We assume that the original limit-cycle solution $\chi$ is only slightly

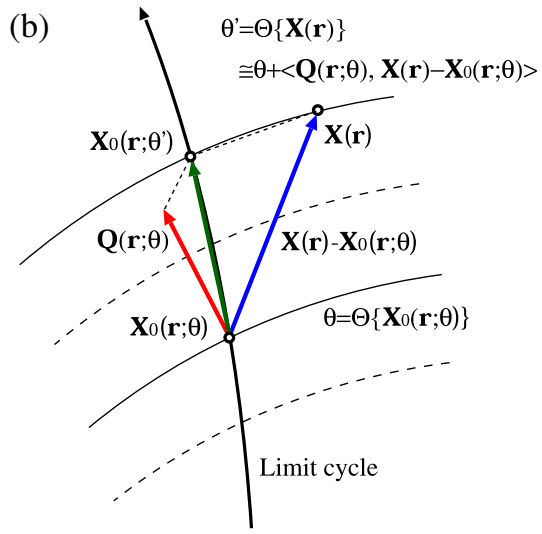

FIG. 6. (a) Isochrons of a limit-cycle solution of a reaction-diffusion system. The same phase value is assigned to the system states (represented by vector fields) that asymptotically converge to the same state on the limit-cycle solution. (b) Linear approximation of the phase near the limit-cycle solution. 
deformed by the perturbation $\mathbf{p}$. If the phase functional $\Theta\{\mathbf{X}(\mathbf{r})\}$ is given, then from Eq. (B8), the phase $\theta(t)=$ $\Theta\{\mathbf{X}(\mathbf{r}, t)\}$ of the perturbed system obeys

$$
\begin{aligned}
\dot{\theta}(t) & =\frac{d}{d t} \Theta\{\mathbf{X}(\mathbf{r}, t)\}=\left\langle\left.\frac{\delta \Theta\{\mathbf{X}(\mathbf{r})\}}{\delta \mathbf{X}(\mathbf{r})}\right|_{\mathbf{X}(\mathbf{r})=\mathbf{X}(\mathbf{r}, t)}, \mathbf{F}[\mathbf{X}(\mathbf{r}, t), \mathbf{r}]\right. \\
& \left.+\mathrm{D} \nabla^{2} \mathbf{X}(\mathbf{r}, t)+\mathbf{p}\{\mathbf{X}(\mathbf{r}, t), \mathbf{r}, t\}\right\rangle \\
= & \omega+\left\langle\left.\frac{\delta \Theta\{\mathbf{X}(\mathbf{r})\}}{\delta \mathbf{X}(\mathbf{r})}\right|_{\mathbf{X}(\mathbf{r})=\mathbf{X}(\mathbf{r}, t)}, \mathbf{p}\{\mathbf{X}(\mathbf{r}, t), \mathbf{r}, t\}\right\rangle . \quad(\mathrm{B} 11)
\end{aligned}
$$

However, this is not a closed equation for $\theta(t)$ because the functional derivative of $\Theta\{\mathbf{X}(\mathbf{r}, t)\}$ and the perturbation $\mathbf{p}\{\mathbf{X}(\mathbf{r}, t), \mathbf{r}, t\}$ still depend on $\mathbf{X}(\mathbf{r}, t)$. Therefore, as in the ODE case, we approximate $\mathbf{X}(\mathbf{r}, t)$ in these terms by $\mathbf{X}_{0}(\mathbf{r} ; \theta)$ on $\chi$, assuming the perturbation $\mathbf{p}\{\mathbf{X}(\mathbf{r}, t), \mathbf{r}, t\}$ to be weak enough so that the system state $\mathbf{X}(\mathbf{r}, t)$ deviates from $\mathbf{X}_{0}(\mathbf{r} ; \theta)$ on $\chi$ only slightly. Then, at the lowest-order approximation, a closed phase equation for $\theta(t)$ can be obtained as

$\dot{\theta}(t) \simeq \omega+\left\langle\left.\frac{\delta \Theta\{\mathbf{X}(\mathbf{r})\}}{\delta \mathbf{X}(\mathbf{r})}\right|_{\mathbf{X}(\mathbf{r})=\mathbf{X}_{0}[\mathbf{r} ; \theta(t)]}, \mathbf{p}\left\{\mathbf{X}_{0}[\mathbf{r} ; \theta(t)], \mathbf{r}, t\right\}\right\rangle$,

which is correct up to $O(\|\mathbf{p}\|)$. By denoting $\mathbf{p}(\theta, \mathbf{r}, t)=$ $\mathbf{p}\left\{\mathbf{X}_{0}(\mathbf{r} ; \theta), \mathbf{r}, t\right\}$ and introducing a phase-sensitivity function

$$
\mathbf{Q}(\mathbf{r} ; \theta)=\left.\frac{\delta \Theta\{\mathbf{X}(\mathbf{r})\}}{\delta \mathbf{X}(\mathbf{r})}\right|_{\mathbf{X}(\mathbf{r})=\mathbf{X}_{0}(\mathbf{r} ; \theta)},
$$

the reduced phase equation (B12) can be concisely written as

$$
\dot{\theta}(t)=\omega+\langle\mathbf{Q}(\mathbf{r} ; \theta), \mathbf{p}(\theta, \mathbf{r}, t)\rangle
$$

at the lowest order in the perturbation. The function $\mathbf{Q}(\mathbf{r} ; \theta)$ is the (functional) gradient of the isochron estimated at $\mathbf{X}(\mathbf{r})=\mathbf{X}_{0}(\mathbf{r} ; \theta)$ on the limit-cycle solution $\chi$ and plays the key role in the present theory.

\section{Linear theory around the limit-cycle solution}

Though we have formally developed a geometric theory of phase reduction for the RD system, it is impossible to obtain $\Theta\{\mathbf{X}(\mathbf{r})\}$ explicitly for general RD systems. However, only the phase-sensitivity function $\mathbf{Q}(\mathbf{r} ; \theta)$ is actually necessary to write down the lowest-order phase equation (B14) for the weakly perturbed RD systems Eq. (B10). We thus try to derive the equation for $\mathbf{Q}(\mathbf{r} ; \theta)$ as in the ODE case, focusing only on the vicinity of the limit cycle $\chi$.

We first note that the phase function $\Theta(\mathbf{X})$ for the ODE can be linearly approximated as $\Theta(\mathbf{X}) \simeq \theta+\mathbf{Z}(\theta)$. $\left[\mathbf{X}-\mathbf{X}_{0}(\theta)\right]$ for the oscillator state $\mathbf{X}$ near $\mathbf{X}_{0}(\theta)$ on $\chi$ using the phase-sensitivity function $\mathbf{Z}(\theta)$ defined in Eq. (A12) (see Appendix A). In a similar spirit, we make an ansatz that the phase $\Theta\{\mathbf{X}(\mathbf{r})\}$ of a state $\mathbf{X}(\mathbf{r})$ of the RD system near the state $\mathbf{X}_{0}(\mathbf{r} ; \theta)$ on the limit cycle $\chi$ can be linearly approximated as

$$
\Theta\{\mathbf{X}(\mathbf{r})\} \simeq \theta+\left\langle\mathbf{Q}(\mathbf{r} ; \theta), \mathbf{X}(\mathbf{r})-\mathbf{X}_{0}(\mathbf{r} ; \theta)\right\rangle,
$$

and we examine whether this ansatz is reasonable. When $\mathbf{X}(\mathbf{r})$ is simply a state on the limit cycle $\chi$ with phase $\theta$, i.e., $\mathbf{X}(\mathbf{r})=\mathbf{X}_{0}(\mathbf{r} ; \theta)$, Eq. (B15) gives $\Theta\left\{\mathbf{X}_{0}(\mathbf{r} ; \theta)\right\}=\theta$. If Eq. (B15) is, furthermore, valid for arbitrary states $\{\mathbf{X}(\mathbf{r})\}$ sufficiently close to the unperturbed state $\mathbf{X}_{0}(\mathbf{r} ; \theta)$ on $\chi$, the function $\mathbf{Q}(\mathbf{r} ; \theta)$ will play the role of the phase-sensitivity function for the RD system. This actually holds by choosing the function $\mathbf{Q}(\mathbf{r} ; \theta)$ appropriately. See Fig. 6(b) for a schematic illustration.

We now derive the equation for $\mathbf{Q}(\mathbf{r} ; \theta)$ by generalizing the argument in Refs. [4,5] for the phase-sensitivity function $\mathbf{Z}(\theta)$ of limit cycles described by ODEs (see also Appendix A). At $t=0$, we prepare an initial state $\mathbf{X}_{0}(\mathbf{r} ; \theta=0)=\mathbf{X}_{0}(\mathbf{r}, t=0)$ on the limit cycle $\chi$ with the phase $\theta=0$ and a slightly perturbed initial state

$$
\mathbf{X}(\mathbf{r}, t=0)=\mathbf{X}_{0}(\mathbf{r} ; \theta=0)+\mathbf{y}(\mathbf{r}, t=0)
$$

near $\mathbf{X}_{0}(\mathbf{r} ; \theta=0)$, where $\mathbf{y}(\mathbf{r}, t=0)$ is a small spatial perturbation given to $\mathbf{X}_{0}(\mathbf{r} ; \theta=0)$. We evolve these two states without applying further perturbations. The linearized equation for $\mathbf{y}(\mathbf{r}, t)=\mathbf{X}(\mathbf{r}, t)-\mathbf{X}_{0}[\mathbf{r} ; \theta(t)]$ can be obtained from Eq. (B1) as

$$
\frac{\partial}{\partial t} \mathbf{y}(\mathbf{r}, t)=\mathrm{J}[\theta(t)] \mathbf{y}(\mathbf{r}, t)+\mathrm{D} \nabla^{2} \mathbf{y}(\mathbf{r}, t),
$$

where $\mathbf{J}(\theta)=\mathbf{J}\left[\mathbf{X}_{0}(\mathbf{r} ; \theta)\right]$ is a Jacobi matrix of $\mathbf{F}$ estimated at $\mathbf{X}=\mathbf{X}_{0}(\mathbf{r} ; \theta)$ on $\chi$. From Eq. (B15), the phase of the unperturbed state $\mathbf{X}_{0}[\mathbf{r} ; \theta(t)]$ is $\theta(t)=\Theta\left\{\mathbf{X}_{0}[\mathbf{r} ; \theta(t)]\right\}=$ $\omega t$, and the phase of the perturbed state $\mathbf{X}(\mathbf{r}, t)$ is given by

$$
\begin{aligned}
\theta^{\prime}(t) & =\Theta\{\mathbf{X}(\mathbf{r}, t)\} \\
& =\Theta\left\{\mathbf{X}_{0}[\mathbf{r} ; \theta(t)]+\mathbf{y}(\mathbf{r}, t)\right\} \\
& \simeq \theta(t)+\langle\mathbf{Q}[\mathbf{r} ; \theta(t)], \mathbf{y}(\mathbf{r}, t)\rangle
\end{aligned}
$$

under the linear approximation. This $\theta^{\prime}(t)$ should also increase with a constant frequency $\omega$ by the definition of the isochron, i.e., $d \theta^{\prime}(t) / d t=\omega$, because no perturbation is given after $t=0$. Therefore, the following equation should hold:

$$
\begin{aligned}
0 & =\frac{d}{d t}\left[\theta^{\prime}(t)-\theta(t)\right]=\frac{\partial}{\partial t}\langle\mathbf{Q}[\mathbf{r} ; \theta(t)], \mathbf{y}(\mathbf{r}, t)\rangle \\
& =\left\langle\frac{\partial}{\partial t} \mathbf{Q}[\mathbf{r} ; \theta(t)], \mathbf{y}(\mathbf{r}, t)\right\rangle+\left\langle\mathbf{Q}[\mathbf{r} ; \theta(t)], \frac{\partial}{\partial t} \mathbf{y}(\mathbf{r}, t)\right\rangle
\end{aligned}
$$

Using Eq. (B17), the last term can be transformed as 


$$
\begin{aligned}
& \left\langle\mathbf{Q}[\mathbf{r} ; \theta(t)], \frac{\partial}{\partial t} \mathbf{y}(\mathbf{r}, t)\right\rangle \\
& \quad=\left\langle\mathbf{Q}[\mathbf{r} ; \theta(t)], \mathrm{J}[\theta(t)] \mathbf{y}(\mathbf{r}, t)+\mathrm{D}^{2} \mathbf{y}(\mathbf{r}, t)\right\rangle \\
& \quad=\left\langle\mathrm{J}[\theta(t)]^{\dagger} \mathbf{Q}[\mathbf{r} ; \theta(t)]+\mathrm{D}^{\dagger} \nabla^{2} \mathbf{Q}[\mathbf{r} ; \theta(t)], \mathbf{y}(\mathbf{r}, t)\right\rangle,
\end{aligned}
$$

where, as usual, partial integration is performed assuming that surface terms vanish or cancel, and $\dagger$ denotes the matrix transpose. Equation (B19) now yields

$$
\begin{aligned}
& \left\langle\frac{\partial}{\partial t} \mathbf{Q}[\mathbf{r} ; \theta(t)]+\mathrm{J}[\theta(t)]^{\dagger} \mathbf{Q}[\mathbf{r} ; \theta(t)]+\mathrm{D}^{\dagger} \nabla^{2} \mathbf{Q}[\mathbf{r} ; \theta(t)], \mathbf{y}(\mathbf{r}, t)\right\rangle \\
& \quad=0,
\end{aligned}
$$

which should hold for any $\mathbf{y}(\mathbf{r}, t)$ evolving from arbitrary $\mathbf{y}(\mathbf{r}, t=0)$. Therefore, $\mathbf{Q}[\mathbf{r} ; \theta(t)]$ should satisfy the following adjoint equation:

$$
\frac{\partial}{\partial t} \mathbf{Q}[\mathbf{r} ; \theta(t)]=-\mathrm{J}[\theta(t)]^{\dagger} \mathbf{Q}[\mathbf{r} ; \theta(t)]-\mathrm{D}^{\dagger} \nabla^{2} \mathbf{Q}[\mathbf{r} ; \theta(t)],
$$

or equivalently, by using the relation $\partial / \partial t=\omega \partial / \partial \theta$ $(\theta=\omega t)$

$$
\omega \frac{\partial}{\partial \theta} \mathbf{Q}(\mathbf{r} ; \theta)=-\mathrm{J}(\theta)^{\dagger} \mathbf{Q}(\mathbf{r} ; \theta)-\mathrm{D}^{\dagger} \nabla^{2} \mathbf{Q}(\mathbf{r} ; \theta),
$$

which we presented as Eq. (3) in Sec. II.

Since this adjoint equation is linear, we also need to normalize $\mathbf{Q}(\mathbf{r} ; \theta)$ appropriately. As in the ODE case, the normalization condition for $\mathbf{Q}(\mathbf{r} ; \theta)$ can be obtained by differentiating the identity $\theta(t)=\Theta\left\{\mathbf{X}_{0}[\mathbf{r} ; \theta(t)]\right\}=\omega t$ by $t$ as

$$
\begin{aligned}
\omega & =\frac{d}{d t} \theta(t)=\frac{d}{d t} \Theta\left\{\mathbf{X}_{0}[\mathbf{r} ; \theta(t)]\right\} \\
& =\left\langle\left.\frac{\delta \Theta\{\mathbf{X}(\mathbf{r})\}}{\delta \mathbf{X}(\mathbf{r})}\right|_{\mathbf{X}(\mathbf{r})=\mathbf{X}_{0}[\mathbf{r} ; \theta(t)]}, \frac{\partial}{\partial t} \mathbf{X}_{0}[\mathbf{r} ; \theta(t)]\right\rangle \\
& =\left\langle\mathbf{Q}[\mathbf{r} ; \theta(t)], \mathbf{F}\left(\mathbf{X}_{0}[\mathbf{r} ; \theta(t)], \mathbf{r}\right)+\mathrm{D}^{2} \mathbf{X}_{0}[\mathbf{r} ; \theta(t)]\right\rangle .
\end{aligned}
$$

Therefore, the following normalization condition should be satisfied:

$$
\omega=\left\langle\mathbf{Q}(\mathbf{r} ; \theta), \mathbf{F}\left[\mathbf{X}_{0}(\mathbf{r} ; \theta), \mathbf{r}\right]+\mathrm{D}^{2} \mathbf{X}_{0}(\mathbf{r} ; \theta)\right\rangle .
$$

Note that this condition can also be expressed, again using the relation $\partial / \partial t=\omega \partial / \partial \theta$, as

$$
\left\langle\mathbf{Q}(\mathbf{r} ; \theta), \frac{\partial}{\partial \theta} \mathbf{X}_{0}(\mathbf{r} ; \theta)\right\rangle=1
$$

which yields the normalization condition Eq. (4) given in Sec. II.
Thus, if the function $\mathbf{Q}(\mathbf{r} ; \theta)$ is the $2 \pi$-periodic solution to Eq. (B23) with the constraint (B26), the approximate phase function Eq. (B15) will satisfy the desired condition Eq. (B19) in the vicinity of $\chi$, and $\mathbf{Q}(\mathbf{r} ; \theta)$ will play the role of the phase-sensitivity function of the limit-cycle solution $\chi$ of the RD system. Note that Eqs. (B23) and (B26) are straightforward generalizations of the conventional adjoint method for the ODE, Eq. (A15), and Eq. (A16). Generally, the function $\mathbf{Q}(\mathbf{r} ; \theta)$ should be calculated numerically by solving the adjoint Eq. (B23) with Eq. (B26). In numerical calculations, it is useful to integrate the adjoint equation (B23) backward in time to avoid numerical overflow with occasional normalization by Eq. (B26), as we explained in Sec. III A.

Once we obtain the frequency $\omega$ and the phasesensitivity function $\mathbf{Q}(\mathbf{r} ; \theta)$, we can write down the approximate phase equation (B14) for a slightly perturbed RD system described by Eq. (B10). Note that the infinitedimensional RD system subjected to weak perturbations is reduced to a single one-dimensional phase equation, which drastically simplifies the analysis of weakly perturbed rhythmic spatiotemporal patterns. As a simple example of this phase reduction theory for RD systems, we analyzed synchronization dynamics of a pair of coupled RD systems exhibiting rhythmic patterns in the previous section, i.e., the circulating pulses, oscillating spots, target waves, and rotating spirals of the FitzHugh-Nagumo model.

\section{APPENDIX C: PHASE-SENSITIVITY FUNCTIONS OF THE TARGET-WAVE SOLUTION}

In Fig. 3, the limit-cycle solution of the FitzHughNagumo (FHN) reaction-diffusion model representing target waves and its phase-sensitivity function are shown only for $\theta=0$. In Figs. 7 and 8, the limit-cycle solution $\mathbf{X}_{0}(\mathbf{r} ; \theta)$ and the phase-sensitivity function $\mathbf{Q}(\mathbf{r} ; \theta)$ are displayed for the whole period of oscillation $(0 \leq \theta<2 \pi)$ with intervals of $\pi / 8$, for the same parameter values as those used in Fig. 3.

We can observe that both the activator $(u)$ and inhibitor (v) components of the phase-sensitivity function $\mathbf{Q}(\mathbf{r} ; \theta)$ are spatially localized at the pacemaker region. As the system undergoes an oscillation and emits a target wave from the pacemaker region, both components of $\mathbf{Q}(\mathbf{r} ; \theta)$ around the pacemaker region oscillate once and become either positive or negative. Because $\mathbf{Q}(\mathbf{r} ; \theta)$ is localized, the perturbations should be given to the pacemaker region to affect the overall phase of the target-wave solution; those applied to other regions have little effect on the phase of the target wave. This is a consequence of the fact that the overall rhythm of the target-wave solution is essentially controlled by the pacemaker region.

In a pair of mutually coupled RD systems exhibiting target waves, the mutual coupling terms are treated as the perturbations. The phase of the target-wave solution in each system is either advanced or retarded when the pacemaker 


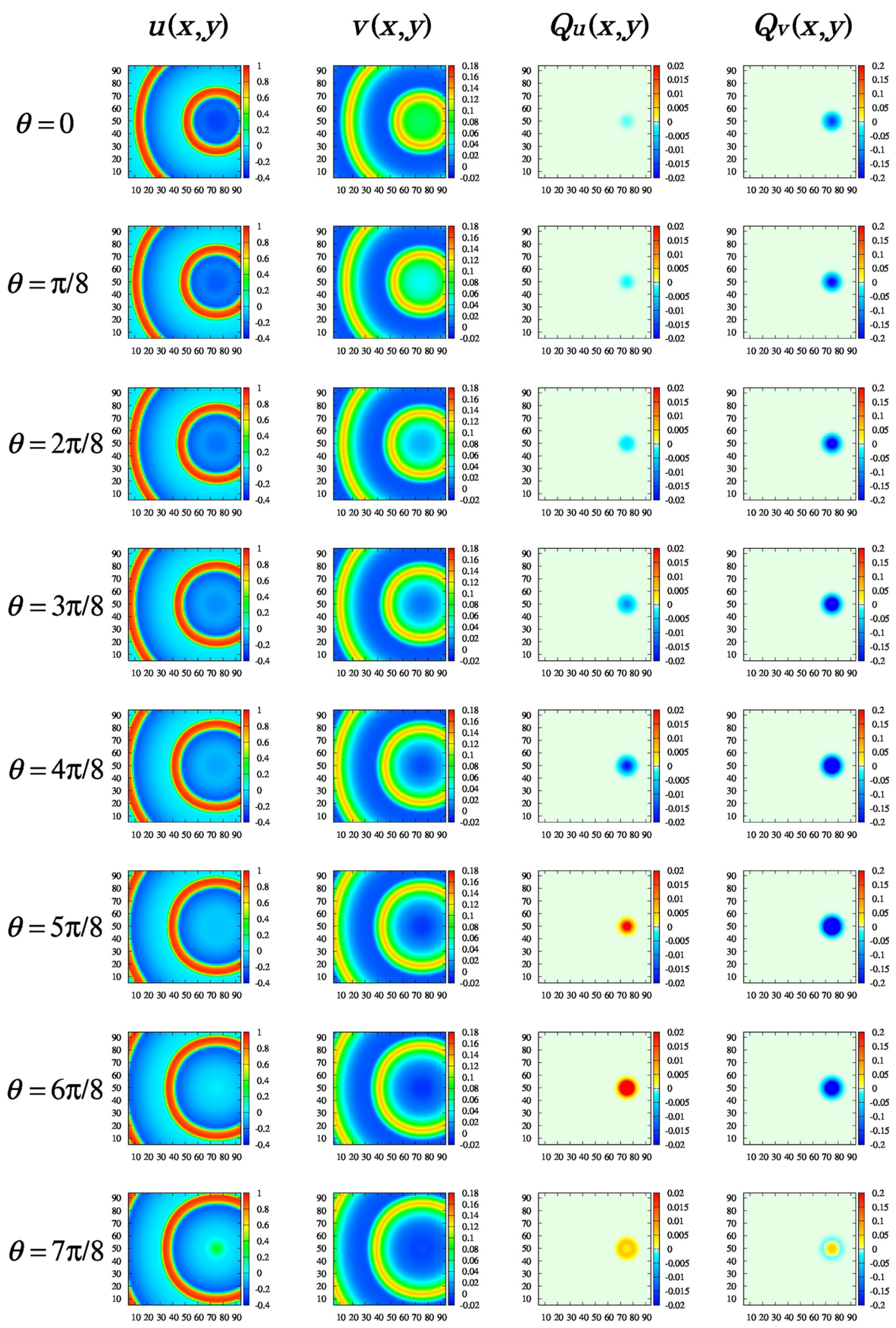

FIG. 7. Limit-cycle solution $\mathbf{X}_{0}(\mathbf{r} ; \theta)$ and phase-sensitivity function $\mathbf{Q}(\mathbf{r} ; \theta)$ of the target wave for $\theta=0, \pi / 8,2 \pi / 8, \ldots, 7 \pi / 8$. First column: Activator $(u)$ component of $\mathbf{X}_{0}(\mathbf{r} ; \theta)$. Second column: Inhibitor $(v)$ component of $\mathbf{X}_{0}(\mathbf{r} ; \theta)$. Third column: $u$ component of $\mathbf{Q}(\mathbf{r} ; \theta)$. Fourth column: $v$ component of $\mathbf{Q}(\mathbf{r} ; \theta)$. The parameters are the same as in Fig. 3. 


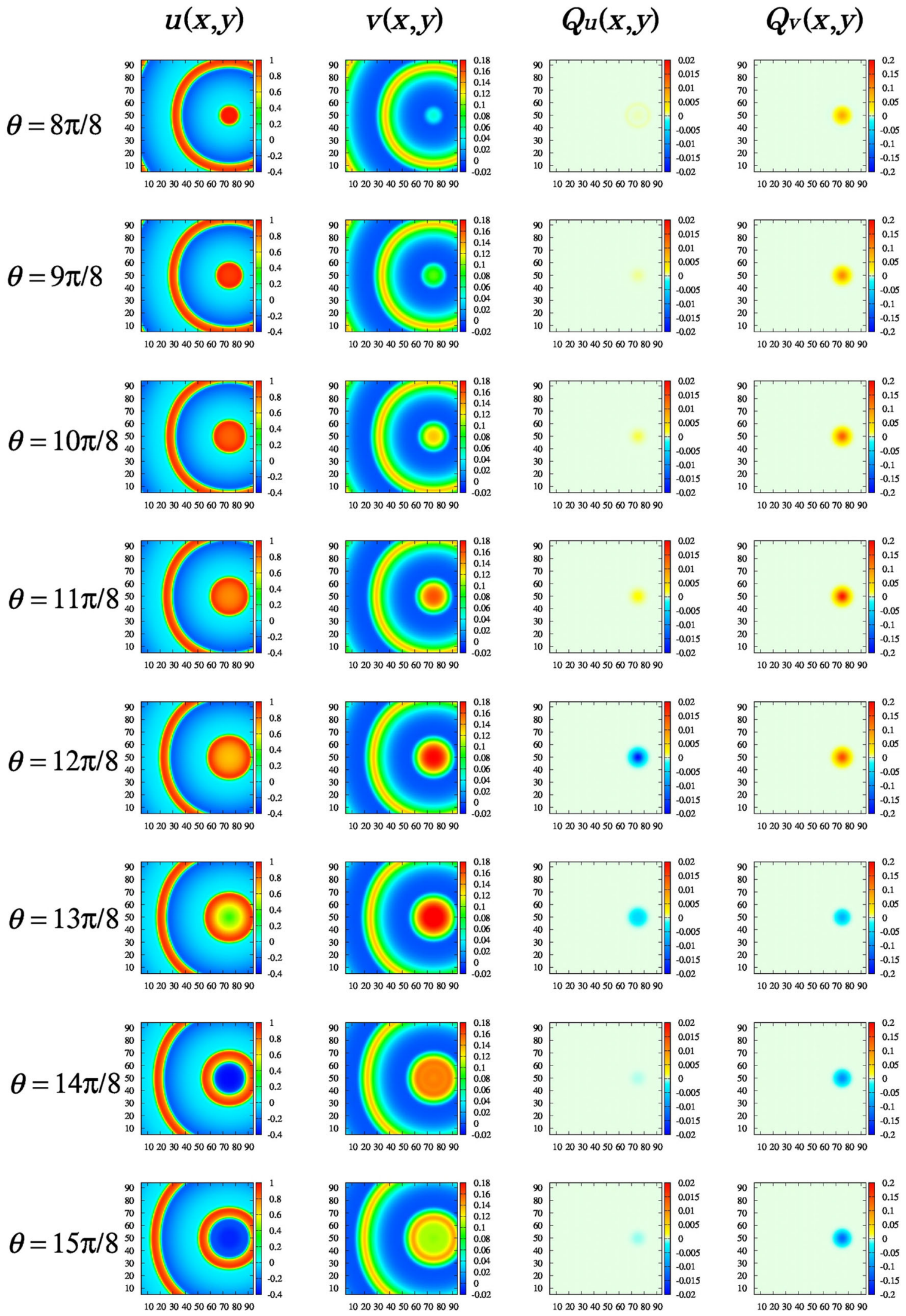

FIG. 8. Limit-cycle solution $\mathbf{X}_{0}(\mathbf{r} ; \theta)$ and phase-sensitivity function $\mathbf{Q}(\mathbf{r} ; \theta)$ of the target wave for $\theta=8 \pi / 8,9 \pi / 8,10 \pi / 8, \ldots, 15 \pi / 8$. First column: Activator $(u)$ component of $\mathbf{X}_{0}(\mathbf{r} ; \theta)$. Second column: Inhibitor $(v)$ component of $\mathbf{X}_{0}(\mathbf{r} ; \theta)$. Third column: $u$ component of $\mathbf{Q}(\mathbf{r} ; \theta)$. Fourth column: $v$ component of $\mathbf{Q}(\mathbf{r} ; \theta)$. The parameters are the same as in Fig. 3 . 
region is perturbed by the target wave of the other system. The net effect of the phase-response property and the mutual coupling leads to either in-phase or antiphase synchronization of the two target waves, which is quantitatively characterized by the function $\Gamma_{a}(\phi)$. As we showed in Sec. III, $\Gamma_{a}(\phi)$ allows both types of synchronization, and one is selected by the initial phase difference between the two RD systems.

\section{APPENDIX D: NONLINEAR PHASE RESPONSE TO STRONG PERTURBATIONS}

The main focus of the present study is to develop a phase-reduction theory for weakly perturbed rhythmic spatiotemporal patterns in the linear regime. We derived the phase-sensitivity function, which quantifies the linear response characteristics of the rhythmic pattern to

(a)

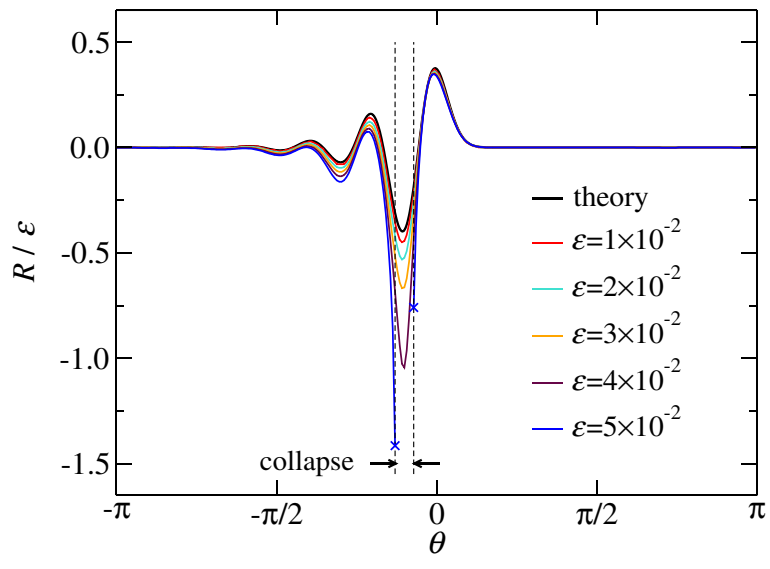

(c)

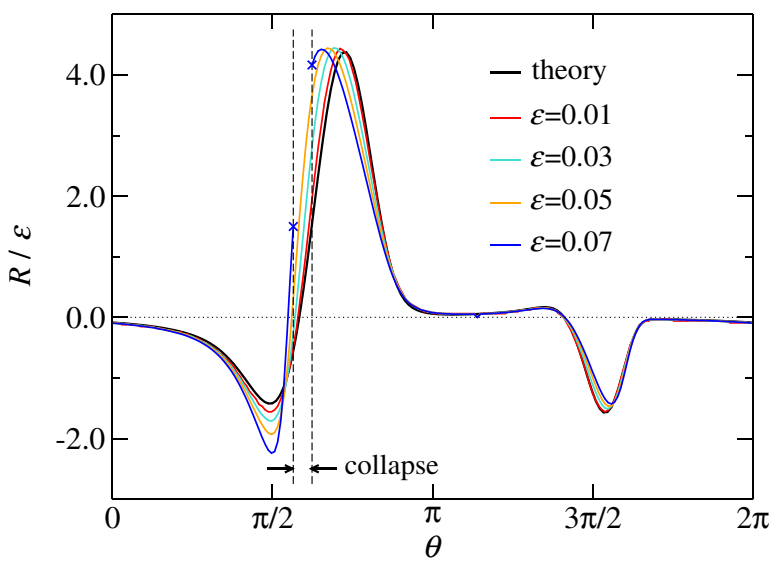

sufficiently weak spatial stimuli. The phase-response curves $R(\theta)$ obtained by direct numerical simulations agreed well with the theoretical curves obtained from the adjoint equation, as shown in Figs. 1-4, when the stimulus intensity $\varepsilon$ is sufficiently small.

As we further increase the stimulus intensity $\varepsilon$ and kick the system state away from the limit cycle, the curvature of the isochron in the infinite-dimensional state space will become non-negligible, and the linear approximation to the isochron, Eq. (2), will be worse. Moreover, if the stimulus intensity becomes too strong, the system state will be kicked out of the basin of the original limit-cycle solution; i.e., the rhythmic spatiotemporal pattern under consideration will collapse, and the definition of the phase itself will become impossible. Though we cannot visualize the isochron in the infinite-dimensional state space of the RD

(b)

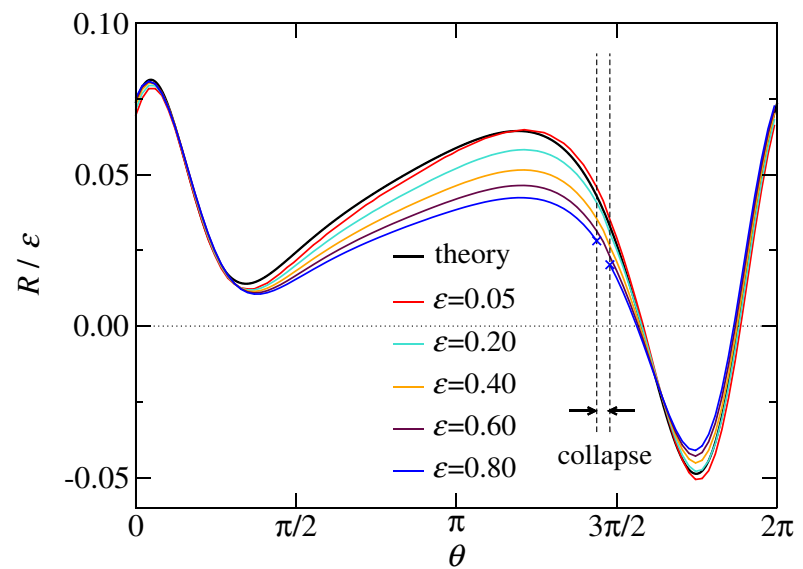

(d)

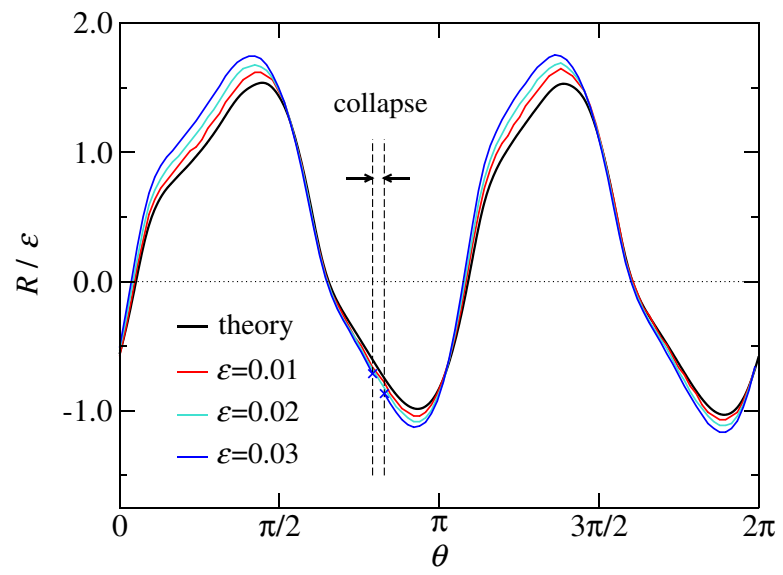

FIG. 9. Phase-response curves of four types of rhythmic spatiotemporal patterns to strong perturbations obtained by direct numerical simulations. Parameters and stimulation patterns are described in Figs. 1-4. (a) Circulating pulse. A bell-shaped perturbation is given to the activator $(u)$ component. When $\varepsilon=5 \times 10^{-2}$, the circulating pulse collapses and the phase response becomes undefined in the interval indicated in the figure. (b) Oscillating spot. A bell-shaped perturbation is given to the activator $(u)$ component. When $\varepsilon=0.8$, the oscillating spot collapses. (c) Target wave. A sinusoidal perturbation is given to the activator $(u)$ component. When $\varepsilon=0.07$, the target wave collapses. (d) Rotating spiral. The checkerboard-like perturbation is given to the activator $(u)$ component. When $\varepsilon=0.03$, the spiral collapses. 
system, these effects are clearly visible in the phaseresponse curves to stronger perturbations obtained by direct numerical simulations.

Figure 9 shows the phase-response curves of the four types of rhythmic spatiotemporal patterns in Figs. 1-4, i.e., the circulating pulse on a ring, oscillating spot, target wave, and rotating spiral, obtained using stronger stimuli. As we increase the stimulus intensity $\varepsilon$, nonlinearity in the phase response (which reflects the curvature of the isochron) becomes prominent, and the normalized phase-response curves start to deviate from the theoretical curve. As $\varepsilon$ exceeds a certain critical value, the original rhythmic spatiotemporal pattern is destroyed and the phase response becomes undefined. The effect of such strong (suprathreshold) stimuli to circulating pulses in excitable media has also been studied by Nomura and Glass in Ref. [21].

For low-dimensional limit-cycle oscillators described by ODEs, numerical methods to calculate the full nonlinear isochrons have been developed [54,55] and higher-order approximations to the isochron have been calculated [56,57]. It may also be possible to develop similar methods for rhythmic spatiotemporal patterns in RD systems, e.g., by representing the spatiotemporal patterns using a finite number of basis functions or by considering higher-order terms in the functional Taylor expansion of the isochron, Eq. (2).

\section{APPENDIX E: FREQUENCY SHIFT OF THE SYNCHRONIZED PATTERNS}

As explained in Sec. II B, the oscillation frequency $\omega+\Gamma(\phi)$ of stably phase-locked rhythmic spatiotemporal patterns can slightly shift from the frequency $\omega$ of the unperturbed single pattern by $\Gamma(\phi)$, where $\phi$ is the stationary phase difference. Table I compares the shift in the frequency measured by direct numerical simulations with the theoretical values $\Gamma(\phi)$ for the four types of rhythmic spatiotemporal patterns shown in Figs. 1-4. For the synchronized circulating pulses in Fig. 1, several stable phase differences (marked as A-D) are possible. For the oscillating spots in Fig. 2, the coupled systems exhibit in-phase synchronization (phase difference $\phi=0$ ) when $L=80$ and antiphase synchronization $(\phi=\pi)$ when $L=120$. For the target waves and spirals in Figs. 3 and 4, both in-phase $(\phi=0)$ and antiphase $(\phi=\pi)$ synchronization are possible depending on the initial conditions. For the in-phase synchronized circulating pulses, target waves, and rotating spirals with the phase difference $\phi=0$, the coupling term vanishes because of symmetry, and correspondingly, $\Gamma(\phi=0)=0$; i.e., no frequency shift occurs [the target waves in Fig. 3 are not symmetrically coupled, and thus $\Gamma(\phi=0) \neq 0$ in this case]. When the two patterns are synchronized in antiphase or with other phase differences, $\Gamma(\phi) \neq 0$ and a small frequency shift occurs. In every case, the theoretical frequency shift $\Gamma(\phi)$ is in
TABLE I. Shift in the frequency of the phase-locked rhythmic spatiotemporal patterns. Theoretical values of the frequency shift $\Gamma(\phi)$, obtained using the theoretical phase-sensitivity function, are compared with those measured by direct numerical simulations. Stable phase differences in the cases B, C, and D of the circulating pulses are obtained by direct numerical simulations.

\begin{tabular}{lccc}
\hline \hline Oscillator & $\begin{array}{c}\text { Stable phase } \\
\text { difference } \phi\end{array}$ & $\begin{array}{c}\text { Theoretical } \\
\text { value } \Gamma(\phi)\end{array}$ & $\begin{array}{c}\text { Numerical } \\
\text { result }\end{array}$ \\
\hline Circulating pulse & $\mathrm{A}(0)$ & 0 & 0 \\
& $\mathrm{~B}(0.5)$ & $-1.3 \times 10^{-4}$ & $-1.4 \times 10^{-4}$ \\
& $\mathrm{C}(1.2)$ & $-1.3 \times 10^{-4}$ & $-1.4 \times 10^{-4}$ \\
& $\mathrm{D}(1.8)$ & $-1.3 \times 10^{-4}$ & $-1.4 \times 10^{-4}$ \\
Oscillating spot & 0 & 0 & 0 \\
$\quad(L=80)$ & & & \\
Oscillating spot & $\pi$ & $1.6 \times 10^{-4}$ & $1.5 \times 10^{-4}$ \\
$\quad(L=120)$ & & & \\
Target wave & 0 & $2.2 \times 10^{-5}$ & $2.4 \times 10^{-5}$ \\
Rotating spiral & $\pi$ & $1.2 \times 10^{-4}$ & $1.2 \times 10^{-4}$ \\
& 0 & 0 & 0 \\
\hline \hline
\end{tabular}

reasonable agreement with the frequency shift obtained by direct numerical simulations.

\section{APPENDIX F: LIST OF VIDEO FILES}

The video files listed below show the dynamics of in-phase and antiphase synchronization in coupled RD systems exhibiting oscillating spots, target waves, and rotating spirals.
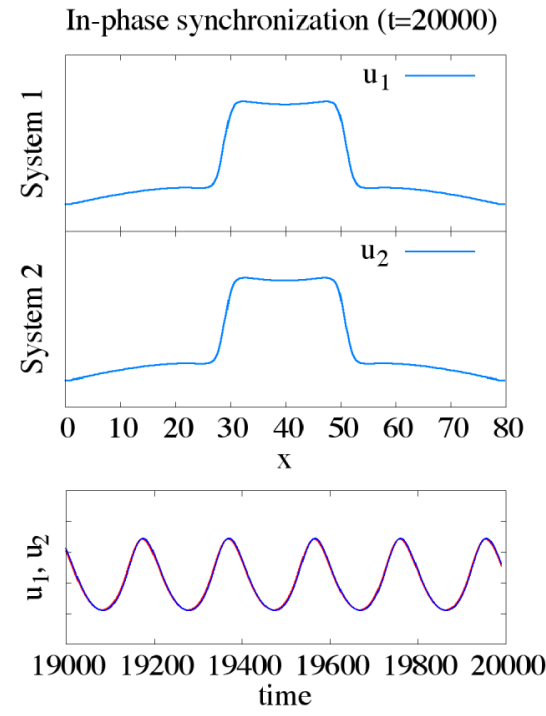

VIDEO 1. In-phase synchronization of oscillating spots in a 1D system of length $L=80$. The parameters are the same as in Fig. 2. The initial phase difference is $\phi(0)=0.616$. The bottom figure shows the evolution of the activator variables $\left(u_{1}, u_{2}\right)$ at the center $(x=L / 2)$ of both systems. 
Anti-phase synchronization ( $\mathrm{t}=20000)$
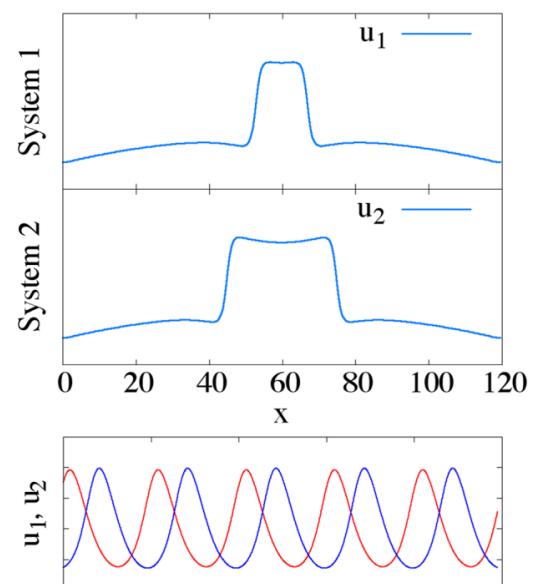

190001920019400196001980020000

time

VIDEO 2. Antiphase synchronization of oscillating spots in a $1 D$ system of length $L=120$. The parameters are the same as in Fig. 2. The initial phase difference is $\phi(0)=1.61$. The bottom figure shows the evolution of the activator variables $\left(u_{1}, u_{2}\right)$ at the center $(x=L / 2)$ of both systems.

In-phase synchronization $(\mathrm{t}=\mathbf{2 5 0 0 0 )}$

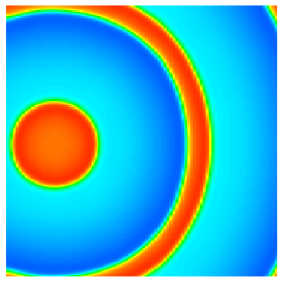

System 2

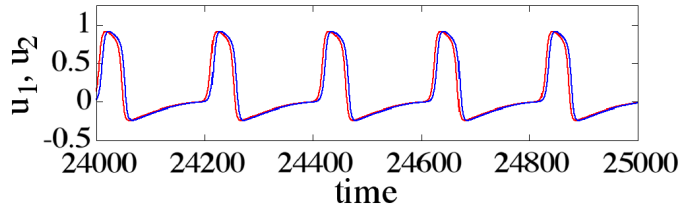

VIDEO 3. In-phase synchronization of target waves. The parameters are the same as in Fig. 3 except for $\mathrm{K}=\operatorname{diag}\left(k=1 \times 10^{-3}, 0\right)$. The initial phase difference is $\phi(0)=1.10$. The bottom figure shows the evolution of the activator variables $u_{1}$ and $u_{2}$ at the corresponding locations, i.e., $\left(x_{1}, y_{1}\right)=(3 L / 4, L / 4)$ of system 1 and $\left(x_{2}, y_{2}\right)=$ $(L / 4, L / 4)$ of system 2 .
Anti-phase synchronization $(\mathrm{t}=25000)$
System 2

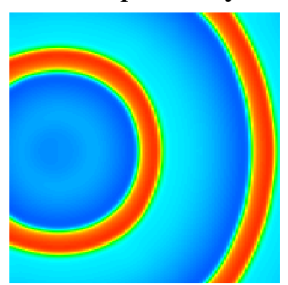

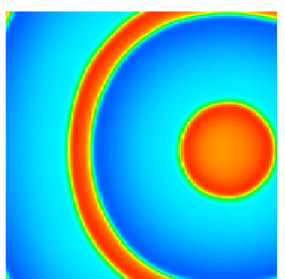

System 1

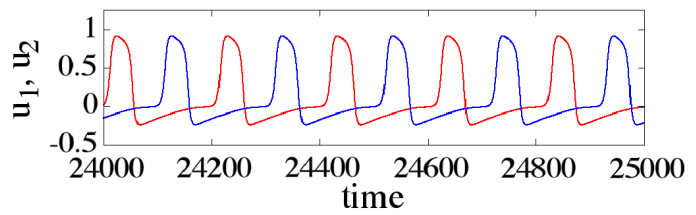

VIDEO 4. Antiphase synchronization of target waves. The parameters are the same as in Fig. 3 except for $\mathrm{K}=\operatorname{diag}\left(k=1 \times 10^{-3}, 0\right)$. The initial phase difference is $\phi(0)=1.16$. The bottom figure shows the evolution of the activator variables $u_{1}$ and $u_{2}$ at the corresponding locations, i.e., $\left(x_{1}, y_{1}\right)=(3 L / 4, L / 4)$ of system 1 and $\left(x_{2}, y_{2}\right)=$ $(L / 4, L / 4)$ of system 2 .

In-phase synchronization $(\mathrm{t}=6000)$

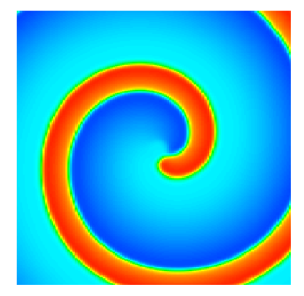

System 2

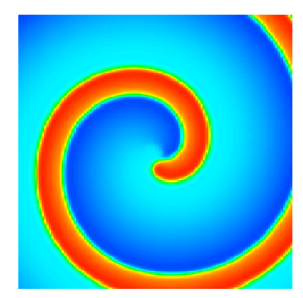

System 1

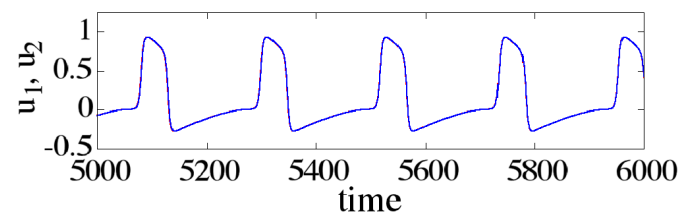

VIDEO 5. In-phase synchronization of rotating spirals. The parameters are the same as in Fig. 4 except for $\mathrm{K}=\operatorname{diag}\left(k=5 \times 10^{-4}, 0\right)$. The initial phase difference is $\phi(0)=1.38$. The bottom figure shows the evolution of the activator variables $\left(u_{1}, u_{2}\right)$ at $(x, y)=(L / 4, L / 4)$ of both systems. 
Anti-phase synchronization $(t=6000)$

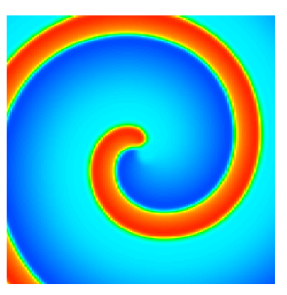

System 2

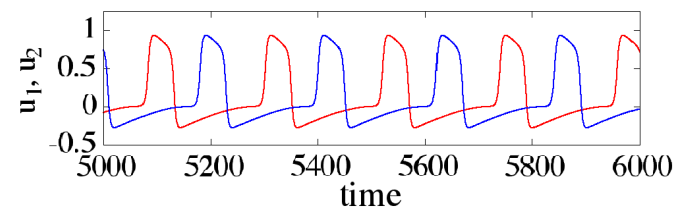

VIDEO 6. Antiphase synchronization of rotating spirals. The parameters are the same as in Fig. 4 except for $\mathrm{K}=\operatorname{diag}\left(k=5 \times 10^{-4}, 0\right)$. The initial phase difference is $\phi(0)=1.44$. The bottom figure shows the evolution of the activator variables $\left(u_{1}, u_{2}\right)$ at $(x, y)=(L / 4, L / 4)$ of both systems.

[1] A. T. Winfree, The Geometry of Biological Time (Springer, Berlin, 1980).

[2] Y. Kuramoto, Chemical Oscillations, Waves, and Turbulence (Springer, Berlin, 1984).

[3] F. C. Hoppensteadt and E. M. Izhikevich, Weakly Connected Neural Networks (Springer, New York, 1997).

[4] E. Brown, J. Moehlis, and P. Holmes, On the Phase Reduction and Response Dynamics of Neural Oscillator Populations, Neural Comput. 16, 673 (2004).

[5] G. B. Ermentrout and D. H. Terman, Mathematical Foundations of Neuroscience (Springer, New York, 2010).

[6] A. Pikovsky, M. Rosenblum, and J. Kurths, Synchronization: A Universal Concept in Nonlinear Sciences (Cambridge University Press, Cambridge, England, 2001).

[7] I. Z. Kiss, Y. Zhai, and J. L. Hudson, Emerging Coherence in a Population of Chemical Oscillators, Science 296, 1676 (2002).

[8] A. F. Taylor, M. R. Tinsley, F. Wang, Z. Huang, and K. Showalter, Dynamical Quorum Sensing and Synchronization in Large Populations of Chemical Oscillators, Science 323, 614 (2009).

[9] M. R. Tinsley, S. Nkomo, and K. Showalter, Chimera and Phase-Cluster States in Populations of Coupled Chemical Oscillators, Nat. Phys. 8, 662 (2012).

[10] I. Z. Kiss, C. G. Rusin, H. Kori, and J. L. Hudson, Engineering Complex Dynamical Structures: Sequential Patterns and Desynchronization, Science 316, 1886 (2007).

[11] J. Moehlis, E. Shea-Brown, and H. Rabitz, Optimal Inputs for Phase Models of Spiking Neurons, J. Comp. Nonlin. Dyn. 1, 358 (2006).

[12] T. Harada, H. A. Tanaka, M. J. Hankins, and I. Z. Kiss, Optimal Waveform for the Entrainment of a Weakly Forced Oscillator, Phys. Rev. Lett. 105, 088301 (2010).
[13] A. Zlotnik, Y. Chen, I. Z. Kiss, H. A. Tanaka, and J. S. Li, Optimal Waveform for Fast Entrainment of Weakly Forced Nonlinear Oscillators, Phys. Rev. Lett. 111, 024102 (2013).

[14] L. Glass, Synchronization and Rhythmic Processes in Physiology, Nature (London) 410, 277 (2001).

[15] L. Glass, Multistable Spatiotemporal Patterns of Cardiac Activity, Proc. Natl. Acad. Sci. U.S.A. 102, 10409 (2005).

[16] G. Bub, A. Shrier, and L. Glass, Spiral Wave Generation in Heterogeneous Excitable Media, Phys. Rev. Lett. 88, 058101 (2002).

[17] M. I. Rabinovich, A. B. Ezersky, and P. D. Weidman, The Dynamics of Patterns (World Scientific, Singapore, 2000).

[18] A. S. Mikhailov and K. Showalter, Control of Waves, Patterns and Turbulence in Chemical Systems, Phys. Rep. 425, 79 (2006).

[19] S. P. Hastings, On the Existence of Homoclinic and Periodic Orbits for the Fitzhugh-Nagumo Equations, Q. J. Math. 27, 123 (1976).

[20] A. Hagberg and E. Meron, Pattern Formation in Nongradient Reaction-Diffusion Systems: The Effects of Front Bifurcations, Nonlinearity 7, 805 (1994).

[21] T. Nomura and L. Glass, Entrainment and Termination of Reentrant Wave Propagation in a Periodically Stimulated Ring of Excitable Media, Phys. Rev. E 53, 6353 (1996).

[22] T. Yanagita, H. Suetani, and K. Aihara, Bifurcation Analysis of Solitary and Synchronized Pulses and Formation of Reentrant Waves in Laterally Coupled Excitable Fibers, Phys. Rev. E 78, 056208 (2008).

[23] Engineering of Chemical Complexity, World Scientific Lecture Notes in Complex Systems Vol. 11, edited by A. S. Mikhailov and G. Ertl (World Scientific Publishing, Singapore, 2013).

[24] J. J. Tyson and J. P. Keener, Singular Perturbation Theory of Traveling Waves in Excitable Media (A Review), Physica D (Amsterdam) 32, 327 (1988).

[25] P. Manneville, Dissipative Structures and Weak Turbulence (Academic Press, San Diego, 1990).

[26] M. Hildebrand, J. Cui, E. Mihaliuk, J. Wang, and K. Showalter, Synchronization of Spatiotemporal Patterns in Locally Coupled Excitable Media, Phys. Rev. E 68, 026205 (2003).

[27] S. Fukushima, S. Nakanishi, K. Fukami, S. I. Sakai, T. Nagai, T. Tada, and Y. Nakato, Observation of Synchronized Spatiotemporal Reaction Waves in Coupled Electrochemical Oscillations of an NDR Type, Electrochem. Comm. 7, 411 (2005).

[28] S. I. Ei, The Motion of Weakly Interacting Pulses in Reaction-Diffusion Systems, J. Dyn. Differ. Equ. 14, 85 (2002).

[29] M. C. Cross and P.C. Hohenberg, Pattern Formation Outside of Equilibrium, Rev. Mod. Phys. 65, 851 (1993).

[30] H. Mori and Y. Kuramoto, Dissipative Structures and Chaos (Springer, Berlin, 1997).

[31] T. Ohta, Pulse Dynamics in a Reaction-Diffusion System, Physica D (Amsterdam) 151, 61 (2001).

[32] G. B. Ermentrout, J.Z. Jalics, and J. E. Rubin, StimulusDriven Traveling Solutions in Continuum Neuronal Models 
with a General Smooth Firing Rate Function, SIAM J. Appl. Math. 70, 3039 (2010).

[33] J. Löber, M. Bär, and H. Engel, Front Propagation in One-Dimensional Spatially Periodic Bistable Media, Phys. Rev. E 86, 066210 (2012).

[34] J. Löber and H. Engel, Controlling the Position of Traveling Waves in Reaction-Diffusion Systems, Phys. Rev. Lett. 112, 148305 (2014).

[35] B. Sandstede, A. Scheel, and C. Wulff, Dynamics of Spiral Waves on Unbounded Domains Using Center-Manifold Reductions, J. Diff. Equ. 141, 122 (1997).

[36] V. N. Biktashev, D. Barkley, and I. V. Biktasheva, Orbital Motion of Spiral Waves in Excitable Media, Phys. Rev. Lett. 104, 058302 (2010).

[37] A. T. Winfree, Biological Rhythms and the Behavior of Populations of Coupled Oscillators, J. Theor. Biol. 16, 15 (1967).

[38] J. Guckenheimer, Isochrons and Phaseless Sets, J. Math. Biol. 1, 259 (1975).

[39] The preliminary result on the phase reduction of circulating pulses was partially presented in our following conference proceedings (not refereed) without a detailed derivation of the phase-reduction theory: H. Nakao, T. Yanagita, and Y. Kawamura, Phase Description of Stable Limit-Cycle Solutions in Reaction-Diffusion Systems, Procedia IUTAM 5, 227 (2012).

[40] The actual advance or delay time of the perturbed pulse is given by $T R(\theta) /(2 \pi)$. For example, the longest advance and delay caused by the bell-shaped stimulus in Fig. 1(b) with $\varepsilon=1.0 \times 10^{-2}$ are 0.33 and -0.35 , respectively.

[41] K. Kotani, I. Yamaguchi, Y. Ogawa, Y. Jimbo, H. Nakao, and G. B. Ermentrout, Adjoint Method Provides Phase Response Functions for Delay-Induced Oscillations, Phys. Rev. Lett. 109, 044101 (2012).

[42] J. N. Teramae and D. Tanaka, Robustness of the NoiseInduced Phase Synchronization in a General Class of Limit Cycle Oscillators, Phys. Rev. Lett. 93, 204103 (2004).

[43] H. Nakao, K. Arai, and Y. Kawamura, Noise-Induced Synchronization and Clustering in Ensembles of Uncoupled Limit-Cycle Oscillators, Phys. Rev. Lett. 98, 184101 (2007).

[44] H. Nakao, K. Arai, K. Nagai, Y. Tsubo, and Y. Kuramoto, Synchrony of Limit-Cycle Oscillators Induced by Random External Impulses, Phys. Rev. E 72, 026220 (2005).
[45] Y. Kawamura, H. Nakao, K. Arai, H. Kori, and Y. Kuramoto, Collective Phase Sensitivity, Phys. Rev. Lett. 101, 024101 (2008).

[46] Y. Kawamura, H. Nakao, and Y. Kuramoto, Collective Phase Description of Globally Coupled Excitable Elements, Phys. Rev. E 84, 046211 (2011).

[47] Y. Kawamura and H. Nakao, Collective Phase Description of Oscillatory Convection, Chaos 23, 043129 (2013).

[48] Y. Kawamura and H. Nakao, Noise-Induced Synchronization of Oscillatory Convection and Its Optimization, Phys. Rev. E 89, 012912 (2014).

[49] V. Novičenko and K. Pyragas, Phase Reduction of Weakly Perturbed Limit Cycle Oscillations in Time-Delay Systems, Physica (Amsterdam) 241D, 1090 (2012).

[50] E. M. Izhikevich, Weakly Connected Quasi-periodic Oscillators, FM Interactions, and Multiplexing in the Brain, SIAM J. Appl. Math. 59, 2193 (1999).

[51] A. Demir, G. Chenjie, and J. Roychowdhury, Phase Equations for Quasi-periodic Oscillators, IEEE/ACM International Conference on Computer-Aided Design (ICCAD) (2010), pp. 292-297, doi: 10.1109/ICCAD.2010.5654185.

[52] Y. Kawamura and H. Nakao, Phase Description of Oscillatory Convection with a Spatially Translational Mode, arXiv:1404.5403.

[53] This is a physical assumption in the present study. Rigorous treatment and explicit computation of the isochrons for RD systems are future research issues.

[54] H. M. Osinga and J. Moehlis, Continuation-Based Computation of Global Isochrons, SIAM J. Appl. Dyn. Syst. 9, 1201 (2010).

[55] A. Mauroy and I. Mezić, On the Use of Fourier Averages to Compute the Global Isochrons of (Quasi)periodic Dynamics, Chaos 22, 033112 (2012).

[56] D. Takeshita and R. Feres, Higher Order Approximation of Isochrons, Nonlinearity 23, 1303 (2010).

[57] O. Suvak and A. Demir, Quadratic Approximations for the Isochrons of Oscillators: A General Theory, Advanced Numerical Methods, and Accurate Phase Computations, IEEE Trans. Comput.-Aided Des. Integr. Circuits Syst. 29, 1215 (2010). 